\title{
Singular separatrix splitting and Melnikov method: An experimental study
}

\author{
Amadeu Delshams and Rafael Ramírez-Ros \\ Departament de Matemàtica Aplicada I \\ Universitat Politècnica de Catalunya \\ Diagonal 647, 08028 Barcelona, Spain
}

November 1997

\begin{abstract}
We consider families of analytic area-preserving maps depending on two parameters: the perturbation strength $\varepsilon$ and the characteristic exponent $h$ of the origin. For $\varepsilon=0$, these maps are integrable with a separatrix to the origin, whereas they asymptote to flows with homoclinic connections as $h \rightarrow 0^{+}$. For fixed $\varepsilon \neq 0$ and small $h$, we show that these connections break up. The area of the lobes of the resultant turnstile is given asymptotically by $\varepsilon \exp \left(-\pi^{2} / h\right) \Theta^{\varepsilon}(h)$, where $\Theta^{\varepsilon}(h)$ is an even Gevrey-1 function such that $\Theta^{\varepsilon}(0) \neq 0$ and the radius of convergence of its Borel transform is $2 \pi^{2}$. As $\varepsilon \rightarrow 0$, the function $\Theta^{\varepsilon}$ tends to an entire function $\Theta^{0}$. This function $\Theta^{0}$ agrees with the one provided by the Melnikov theory, which cannot be applied directly, due to the exponentially small size of the lobe area with respect to $h$.

These results are supported by detailed numerical computations; we use an expensive multiple-precision arithmetic and expand the local invariant curves up to very high order.
\end{abstract}

Keywords: Area-preserving map, singular separatrix splitting, Melnikov method, numerical experiments

AMS Subject Classification (1991): 34C37,34E05,34E15,65L12

\section{Introduction}

\section{The problem}

In this paper, we consider the following family of planar standard-like maps

$$
F(x, y)=\left(y,-x+U^{\prime}(y)\right), \quad U(y)=\mu_{0} \log \left(1+y^{2}\right)+\varepsilon V(y),
$$


where $V(y)=\sum_{n \geq 1} V_{n} y^{2 n}$ is an even entire function.

Provided that $\mu_{0}+V_{1} \varepsilon>1$, the origin $O=(0,0)$ is a hyperbolic fixed point with $\operatorname{Spec}[\mathrm{d} F(O)]=\{\exp ( \pm h)\}$, and its characteristic exponent $h>0$ is given by

$$
\cosh h=\mu_{0}+V_{1} \varepsilon
$$

Moreover, when $\varepsilon$ vanishes, $F$ becomes integrable with a separatrix to the origin. Thus, the map $F$ can be considered as a perturbation of an integrable map, $\varepsilon$ being the perturbation strength. These two parameters, $h>0$ and $\varepsilon$, will be considered the intrinsic parameters of the map $F$ under study.

Our goal is to show that for $\varepsilon \neq 0$ and for a general perturbation, the separatrix splits and exactly two (transverse) primary homoclinic points, $z^{+}$and $z^{-}$, appear on the quadrant $\{x, y>0\}$. By primary homoclinic orbits we mean that these orbits persist for all $\varepsilon$ small enough.

The pieces of the perturbed invariant curves between the points $z^{ \pm}$enclose a region called lobe. Our measure of the splitting size will be the area $A$ of this lobe, see figure 2 . This lobe area is a homoclinic symplectic invariant, that is, it does not depend on the symplectic coordinates used, and all the lobes have the same area. Lobe areas also measure the flux along the homoclinic tangle, which is related to the study of transport [MMP84, MMP87, Mei92].

Both parameters, $h$ and $\varepsilon>0$, will be small 'enough', but the exact interpretation of this sentence is crucial for understanding the different kinds of results to be presented. Specifically, we are going to deal with the following situations:

1. The regular case: fixed $h>0$, and $\varepsilon \rightarrow 0$.

2. The singular case: $h \rightarrow 0^{+}$. In its turn this case subdivides in two sub-cases:

(a) The non-perturbative case: $\varepsilon$ fixed and $h \rightarrow 0^{+}$.

(b) The perturbative case: $\varepsilon=\mathrm{o}\left(h^{p}\right)$ and $h \rightarrow 0^{+}$, for some $p \geq 0$.

Both analytical and numerical results for the splitting of separatrices are obtained. The analytical results are expressed in terms of the Melnikov potential of the problem, which gives explicit formulae for our map. This is the reason for our choice of the map above as a model for this paper, instead of more celebrated maps like the Hénon map or the standard map.

The name "singular" for the case $h \rightarrow 0^{+}$, is due to the fact that the lobe areas are exponentially small in $h$. The measure of such small quantities requires a very careful treatment, both from a numerical and an analytical point of view.

\section{Outline of results}

In the regular case, for $0<|\varepsilon|<\varepsilon^{*}(h)=\mathrm{o}\left(\exp \left(-\pi^{2} / h\right)\right)$, the discrete version of the usual Melnikov method [DR96, DR97b] ensures the existence of two transverse, primary homoclinic orbits, and provides the first order approximation, in the perturbation 
strength $\varepsilon$, of the lobe area:

$$
A=\varepsilon A_{\text {Mel }}+\mathrm{O}\left(\varepsilon^{2}\right), \quad A_{\text {Mel }}=\mathrm{e}^{-\pi^{2} / h}\left[\Theta^{0}(h)+\mathrm{O}\left(\mathrm{e}^{-2 \pi^{2} / h}\right)\right],
$$

where $\Theta^{0}(h)=\sum_{n>0} \Theta_{n}^{0} h^{2 n}$ is an even entire function. If $V(y)$ is a polynomial, $\Theta^{0}(h)$ can be explicitly computed in a finite number of steps. For instance, $\Theta^{0}(h)=8 \pi^{2} \gamma^{2} h^{-2}$ for $V^{\prime}(y)=y$, and $\Theta^{0}(h)=\frac{8}{3} \pi^{2} \gamma^{4} h^{-2}\left[1+\pi^{2} h^{-2}\right]$ for $V^{\prime}(y)=y^{3}$. The non-polynomial case is harder, although some closed formulae can be obtained. In particular, $\Theta^{0}(0)=$ $\Theta_{0}^{0}=8 \pi \hat{V}(2 \pi)$, where $\widehat{V}(\xi)=\sum_{n \geq 1} V_{n} \xi^{2 n-1} /(2 n-1)$ ! is the Borel transform of $V(y)$.

In the singular case, the result above cannot be applied, since it requires $\varepsilon$ to be exponentially small in $h$. There are, however, a couple of analytical results that hold.

In the non-perturbative case, under the assumption

$$
\left(V_{1}+2 V_{2}\right) \varepsilon<1
$$

there exist homoclinic orbits for $h>0$ small enough, and an exponentially small in $h>0$ upper bound is provided for the lobe area.

In the perturbative case $\varepsilon=\mathrm{o}\left(h^{p}\right)$, with $p>6$, under the assumption $\widehat{V}(2 \pi) \neq 0$, the existence of two transverse, primary homoclinic orbits in the first quadrant is proved, and an asymptotic expression for the area lobe is given:

$$
A=\varepsilon \mathrm{e}^{-\pi^{2} / h}\left[8 \pi \hat{V}(2 \pi)+\mathrm{O}\left(h^{2}\right)\right] \quad\left(h \rightarrow 0^{+}\right) .
$$

Most of these analytical results are spread out over several recent papers of the authors [DR96, DR97b, DR97a]. For the convenience of the reader, we have collected in the present paper the main ideas.

The heart of this paper is dedicated to study numerically the situations not covered by the analytical results for the singular case. The numerical experiments have been performed for the simplest even perturbed potentials, that is, for the linear perturbation $\varepsilon V^{\prime}(y)=\varepsilon y$, and the cubic one $\varepsilon V^{\prime}(y)=\varepsilon y^{3}$.

In the non-perturbative case, the following asymptotic expansion for the lobe area $A$ is numerically established

$$
A \sim \varepsilon \mathrm{e}^{-\pi^{2} / h} \sum_{n \geq 0} \Theta_{n}^{\varepsilon} h^{2 n} \quad\left(h \rightarrow 0^{+}, \varepsilon \text { fixed }\right) .
$$

The sign $\sim$ means that the series $\sum_{n>0} \Theta_{n}^{\varepsilon} h^{2 n}$ is an asymptote, that is, if one retains a finite number of the first successive terms, the error has the order of the first missing term:

$$
\left|A-\varepsilon \mathrm{e}^{-\pi^{2} / h} \sum_{n=0}^{N} \Theta_{n}^{\varepsilon} h^{2 n}\right|=\mathrm{O}\left(\varepsilon h^{2 N+2} \mathrm{e}^{-\pi^{2} / h}\right) .
$$

The coefficients $\Theta_{n}^{\varepsilon}$ are real numbers such that

$$
\Theta_{n}^{\varepsilon}=(2 n) !\left(2 \pi^{2}\right)^{-2 n}(2 n)^{4}\left[\Xi_{\infty}^{\varepsilon}+\mathrm{O}\left(n^{-1}\right)\right] \quad(n \rightarrow+\infty),
$$


for some non-zero constant $\Xi_{\infty}^{\varepsilon}$. In particular, $\sum_{n} \Theta_{n}^{\varepsilon} h^{2 n}$ is divergent for all $h \neq 0$, but its Borel transform $\widehat{\Theta^{\varepsilon}}(h)=\sum_{n} \Theta_{n}^{\varepsilon} \xi^{2 n-1} /(2 n-1)$ ! is convergent for $|\xi|<2 \pi^{2}$. This implies that the function $\Theta^{\varepsilon}(h) \sim \varepsilon^{-1} \exp \left(\pi^{2} / h\right) A$ is Gevrey-1 of type $\rho=1 / 2 \pi^{2}$. Let us recall that a function $f(x) \sim \sum_{n>0} f_{n} x^{n}$ is said to be Gevrey-r of type $\rho$ if there are positive constants $C, \alpha>0$ such that $\left|f_{n}\right| \leq C \rho^{r n} \Gamma(r n+\alpha)$, where $\Gamma(z)$ stands for the Gamma function. (We follow the notations of [RS96].)

In the perturbative case, we study the behavior of the objects $\Theta^{\varepsilon}(h), \Theta_{n}^{\varepsilon}, \Xi_{\infty}^{\varepsilon}$, checking that all of them tend to well-defined limits, as $\varepsilon \rightarrow 0$. (That is, for $\varepsilon=\mathrm{o}(1)$. In the notation $\varepsilon=\mathrm{o}\left(h^{p}\right)$, this means that $p=0$.)

First, the function $\Theta^{\varepsilon}(h)$ tends to the Melnikov prediction $\Theta^{0}(h)$ when the perturbation strength $\varepsilon$ tends to zero; more precisely,

$$
\Theta^{\varepsilon}(h)=\Theta^{0}(h)+\mathrm{O}(\varepsilon), \quad \text { uniformly in } h \in(0,1] .
$$

The coefficients $\Theta_{n}^{\varepsilon}$ of the Gevrey series for $\Theta^{\varepsilon}(h)$ also converge to the Taylor coefficients $\Theta_{n}^{0}$ of the entire function $\Theta^{0}(h)$. ( For example, $\Theta_{0}^{\varepsilon}=8 \pi \widehat{V}(2 \pi)+\mathrm{O}(\varepsilon)$.) Obviously, this convergence cannot be uniform in the index $n$, since

$$
\lim _{n \rightarrow+\infty}\left|\Theta_{n}^{\varepsilon}\right|= \begin{cases}0 & \text { if } \varepsilon=0 \\ +\infty & \text { otherwise. }\end{cases}
$$

Finally, $\lim _{\varepsilon \rightarrow 0} \Xi_{\infty}^{\varepsilon}=0$, since $\Xi_{\infty}^{\varepsilon}$ quantifies the growth of the coefficients $\Theta_{n}^{\varepsilon}$, and $\Theta_{n}^{0}=\lim _{\varepsilon \rightarrow 0} \Theta_{n}^{\varepsilon}$ gives a decreasing sequence. In fact, one has

$$
\Xi_{\infty}^{\varepsilon}=\varepsilon \Xi_{\infty}^{0}+\mathrm{O}\left(\varepsilon^{2}\right), \quad \text { where } \quad \Xi_{\infty}^{0}= \begin{cases}-12 \pi^{-4} & \text { if } V^{\prime}(y)=y \\ -16 / 3 & \text { if } V^{\prime}(y)=y^{3}\end{cases}
$$

\section{Relation to other work}

By now, there is a well-developed literature on singular perturbations for maps. Results showing that the splitting size is exponentially small in the characteristic exponent $h$ have been obtained by many authors. For the sake of brevity, we review results concerning analytic area-preserving maps, both from a theoretical and a numerical point of view. For a review of the results concerning flows, we refer to [DS97, DRS97], and the references therein.

The first relevant results are exponentially small upper bounds of the splitting size for analytic area-preserving maps with a weakly hyperbolic fixed point and homoclinic points to it [Nei84, FS90, Fon95, FS96, Gel96]. Roughly speaking, in these papers it is proved that the maps asymptote to a Hamiltonian flow with a separatrix when the characteristic exponent $h$ tends to zero. Then, the splitting size is $\mathrm{O}(\exp (-\beta / h))$, for any positive constant $\beta$ smaller than $2 \pi d, d$ being the analyticity width of the separatrix of the limit flow. No more general results are known. In order to compare this result with the next ones, it is convenient to formulate it as

$$
\text { splitting size }=\mathrm{e}^{-\beta / h} \Theta(h), \quad \Theta(h) \text { bounded when } h \rightarrow 0^{+} \text {. }
$$


The next step was the attainment of exponentially small asymptotic formulae in some standard-like maps, by V. Lazutkin and co-workers [Laz84, LST89, GLT91], see also [HM93, Sur94, Tre96]. For instance, regarding the standard map and the Hénon map, in these works it is claimed that the splitting has an asymptotic behavior of the form $\omega_{0} h^{\gamma} \exp (-\beta / h)$, for some constants $\omega_{0} \neq 0, \beta>0$, and $\gamma$, that is,

$$
\text { splitting size }=h^{\gamma} \mathrm{e}^{-\beta / h} \Theta(h), \quad \Theta(h) \text { continuous at } h=0 \text { and } \Theta(0) \neq 0 .
$$

The constant $\omega_{0}=\Theta(0)$ is defined by means of a nonlinear parameterless problem which only can be solved numerically, $\gamma$ is obtained by linearization about the separatrix in the complex plane, and $\beta=2 \pi d$, where $d$ is again the analyticity width of the unperturbed separatrix. A complete proof of these asymptotic formulae has not been published yet, but there is little doubt about its validity. It should be noted that there exist examples where a formula like (1.2) cannot hold, because the splitting behaves asymptotically like $\omega_{0} h^{\gamma} \exp (-\beta / h) \cos (\alpha / h)$ with $\alpha \neq 0$, see [GLT91, SMH91]. The maps here considered do not fall into this class.

The strongest analytical results on the regularity of the function $\Theta(h)$ were contained in [GLS94, Che95, Nik95], where it is stated (again without proofs) that

$$
\text { splitting size }=h^{\gamma} \mathrm{e}^{-\beta / h} \Theta(h), \quad \Theta(h) \text { smooth at } h=0 \text { and } \Theta(0) \neq 0,
$$

for the standard map [GLS94], the Hénon map [Che95], and the twist map [Nik95]. All these works contains formulae like

$$
\omega[\mathcal{O}] \sim h^{\gamma} \mathrm{e}^{-\beta / h} \sum_{n \geq 0} \omega_{n} h^{2 n}
$$

where $\omega[\mathcal{O}]$ stands for the Lazutkin's homoclinic invariant introduced in [GLT91] for some distinguished symmetric homoclinic orbit $\mathcal{O}$. Only a few coefficients $\omega_{n}$ were explicitly computed in these works: the first five coefficients in [GLS94], the first three in [Nik95] and just two in [Che95]. Then, a natural question appears: Which is the growth rate of the coefficients $\omega_{n}$ when $n \rightarrow+\infty$ ? Or equivalently, is $\Theta(h)$ somewhat stronger than smooth?

A numerical answer involves the computation of many of such coefficients. Recent numerical experiments performed by C. Simó suggest that the asymptotic series $\sum_{n \geq 0} \omega_{n} h^{2 n}$ are divergent, although their Borel transforms are convergent, that is,

$$
\text { splitting size }=h^{\gamma} \mathrm{e}^{-\beta / h} \Theta(h), \quad \Theta(h) \text { Gevrey-1 at } h=0 \text { and } \Theta(0) \neq 0 .
$$

Our numerical results fall just into this class, with the area $A$ as our measure of the splitting size, and the coefficients $\Theta_{n}^{\varepsilon}$ playing the rôle of $\omega_{n}$. The computation of $\omega_{n}$ for relatively large values of $n$ (namely, up to $n=100$ ), requires the use of an expensive multiple-precision arithmetic, so that these experiments are on the edge of the current computer possibilities. Therefore, further numerical results improving these ones are unlikely to appear in a near future. 
Regarding rigorous results, to the best of our knowledge, the paper [DR97a] is the only place where a behavior like (1.2) has been rigorously proved for some areapreserving maps. This makes evident that experimental studies are much more advanced than analytical ones. However, numerical results of the form (1.4) open the door to new techniques, like resurgence tools, that have been already applied to the rapidly forced pendulum [Sau95], and may be successful to fill this gap between analytical and numerical results.

\section{Outline of the computations}

The area of the lobes of the turnstile created when the separatrices split is computed using the MacKay-Meiss-Percival action principle [MMP84, Eas91], in which the lobe area is interpreted as a difference of actions. The numerical computation of such exponentially small lobe areas with arbitrary precision forces us to:

- use an expensive multiple-precision arithmetic,

- expand the invariant curves up to an optimal order, which is very large,

- take the greatest advantage of symmetries and/or reversors.

Clearly, the first item is unavoidable, due to the strong cancellation produced when subtracting the (exponentially close) actions, and also due to the requirement of arbitrary precision in the final result. The second item is intended to take the initial iterates far enough of the weakly hyperbolic point so that the homoclinic points $z^{ \pm}$can be attained in (relatively) few iterations: we are able to find the (optimal) order which minimizes the computer time. This optimal choice of order avoids an undesirable accumulation of rounding errors due to the large number of operations. Finally, the third item is crucial to overcome certain stability problems. Those algorithms for computing homoclinic points that do not take into account symmetries and/or reversors (if they exist, of course) have condition numbers inversely proportional to the splitting size, see for instance [BK97, pag. 1218]. Therefore, they would be exponentially ill-conditioned for our singular maps!

We have improved the methods used in [LM96] to compute lobe areas. In that paper a similar problem was studied, but the invariant curves were developed only to first (linear) order and a standard double-precision arithmetic was used. Due to this, the computations in [LM96] only gave accurate results for lobe areas $A \gg 10^{-14}$, that is, for characteristic exponents $h$ not smaller than $1 / 3$. In this work, we have been able to compute lobe areas less than $10^{-4200}$ (that is, we have arrived up to $h=0.001$ ), with a relative error less than $10^{-900}$. The computation for such extreme cases takes between two and three days on a Pentium 200 under a Linux operative system, depending on the potential $V(y)$. More than 5200 decimal digits in the arithmetic and 1300 coefficients in the Taylor expansion of the invariant curves have been needed for these accurate computations. 
So far, and to the best of our knowledge, the most refined (published) experiments about singular splittings for maps were those of [FS90], where splittings of order $10^{-200}$ were numerically computed following the above-mentioned items. Other experiments with multiple-precision arithmetic are contained in [FS96], but only order one (that is, linear) expansions of the invariant curves were used in that paper. In [BO93] a quadruple-precision together with high order expansion were used to study the rapidly forced pendulum.

\section{Outline of the paper}

The rest of the paper is devoted to explain how our results are be obtained. In the next section, the model is introduced. In section 3 , the regular case $\varepsilon \rightarrow 0$ and $h$ fixed is discussed. We review how to compute the $\mathrm{O}(\varepsilon)$-approximation of the lobe area using the discrete version of the Melnikov method. In particular, the entire function $\Theta^{0}(h)$ is introduced. Section 4 is devoted to the singular limit $h \rightarrow 0^{+}$. The asymptotic behavior of $\Theta^{\varepsilon}(h)$ is studied and the connection with the Melnikov theory is drawn. The results in this section are the heart of the paper. In section 5, the algorithm used to compute lobe areas with an arbitrary accuracy is described. This is the key tool in this work. The numerical calculations are complicated by problems of stability, precision and computer time, so we provide sufficient detail to show how these problems can be overcome. Finally, further numerical experiments related to singular separatrix splittings for maps are proposed in section 6 . They will the subject of future research.

\section{The model}

The family of standard-like maps under study is given by

$$
F(x, y)=\left(y,-x+U^{\prime}(y)\right), \quad U(y)=\mu_{0} \log \left(1+y^{2}\right)+\varepsilon V(y),
$$

where $V(y)=\sum_{n \geq 1} V_{n} y^{2 n}$ is an even entire function. For

$$
\mu:=\mu_{0}+\varepsilon V_{1}>1,
$$

the origin $O=(0,0)$ is a hyperbolic fixed point with $\operatorname{Spec}[\mathrm{d} F(O)]=\left\{\mathrm{e}^{ \pm h}\right\}$, where the characteristic exponent $h>0$ is determined by $\cosh h=\mu$.

We will consider the characteristic exponent $h$ and the perturbation strength $\varepsilon$ as the intrinsic parameters of our model. Accordingly, for every $h>0$ and every real $\varepsilon$, we rewrite the map (2.1) in the form

$$
\begin{array}{ll}
F(x, y)=\left(y,-x+U^{\prime}(y)\right), & U(y)=U_{0}(y)+\varepsilon U_{1}(y), \\
U_{0}(y)=\mu \log \left(1+y^{2}\right), & U_{1}(y)=V(y)-V_{1} \log \left(1+y^{2}\right) .
\end{array}
$$

From now on, the subscript "0" will denote an unperturbed quantity, that is, $\varepsilon=0$, and the following notations will be used without further comment:

$$
\mu=\cosh h, \quad \gamma=\sinh h, \quad \lambda=\mathrm{e}^{h} .
$$




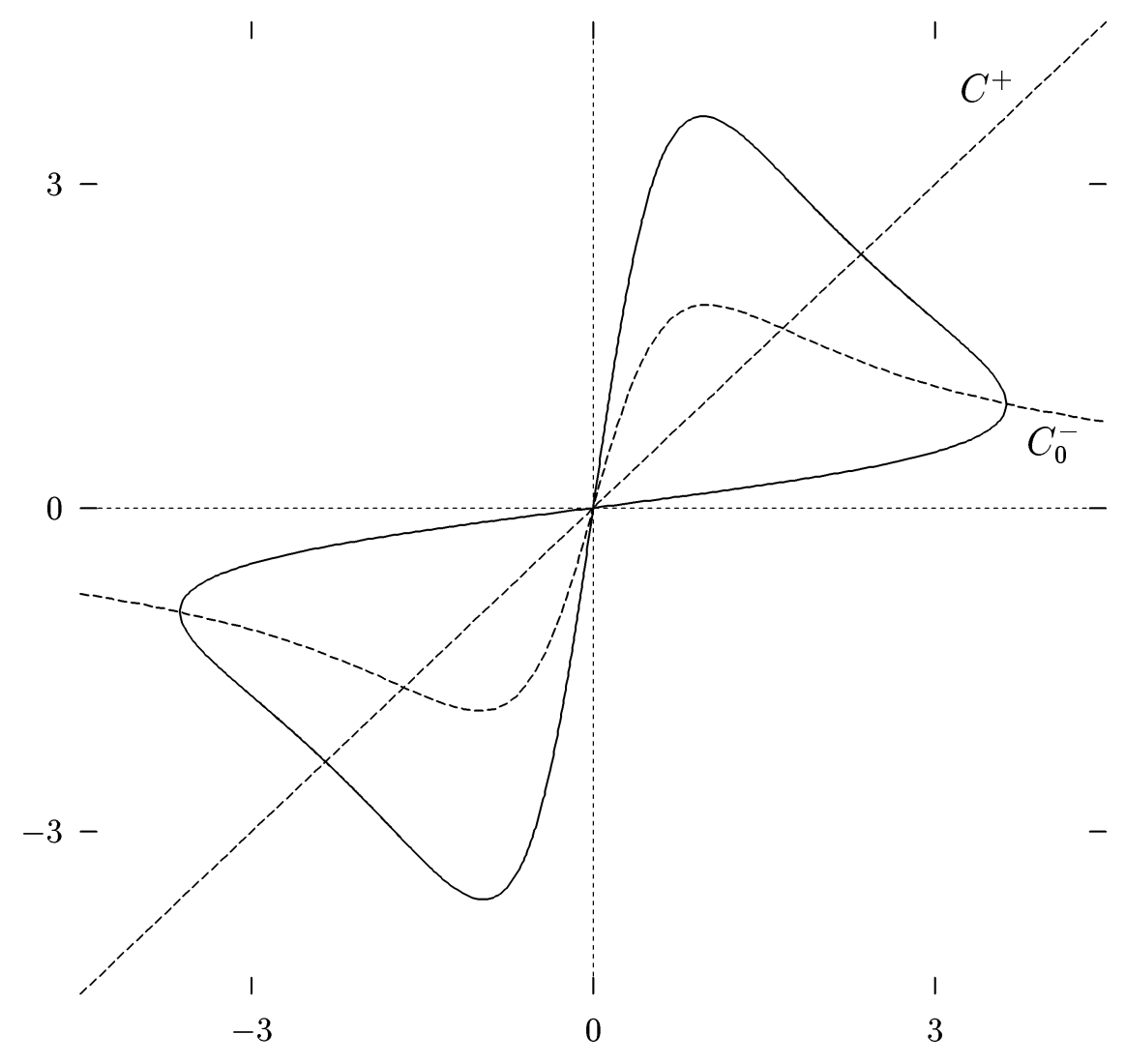

Figure 1: The zero level of $I_{0}$ for $h=2$.

\section{The unperturbed model}

Setting $\varepsilon=0$ in (2.2), we obtain the so-called McMillan map [McM71]

$$
F_{0}(x, y)=\left(y,-x+U_{0}^{\prime}(y)\right)=\left(y,-x+\frac{2 \mu y}{1+y^{2}}\right),
$$

which is an integrable exact map, with a polynomial first integral given by

$$
I_{0}(x, y)=x^{2}-2 \mu x y+y^{2}+x^{2} y^{2} .
$$

The phase space associated to $F_{0}$ is rather simple, since it is foliated by the level curves of the first integral $I_{0}$, which are symmetric with respect to the origin. As $\mu>1$, the zero level of $I_{0}$ is a lemniscate, whose loops are separatrices to the origin (see figure 1). From now on, we will concentrate on the separatrix $\Lambda$ in the quadrant $\{x, y>0\}$, which can be parameterized by

$$
z_{0}(t)=\left(x_{0}(t), y_{0}(t)\right)=\left(\xi_{0}(t-h / 2), \xi_{0}(t+h / 2)\right), \quad \xi_{0}(t)=\gamma \operatorname{sech} t .
$$

This parameterization is called natural since $F_{0}\left(z_{0}(t)\right)=z_{0}(t+h)$, a fact that can be checked simply by noting that $\xi_{0}(t)$ is a homoclinic solution of the difference equation

$$
\xi_{0}(t+h)+\xi_{0}(t-h)=U_{0}^{\prime}\left(\xi_{0}(t)\right)
$$




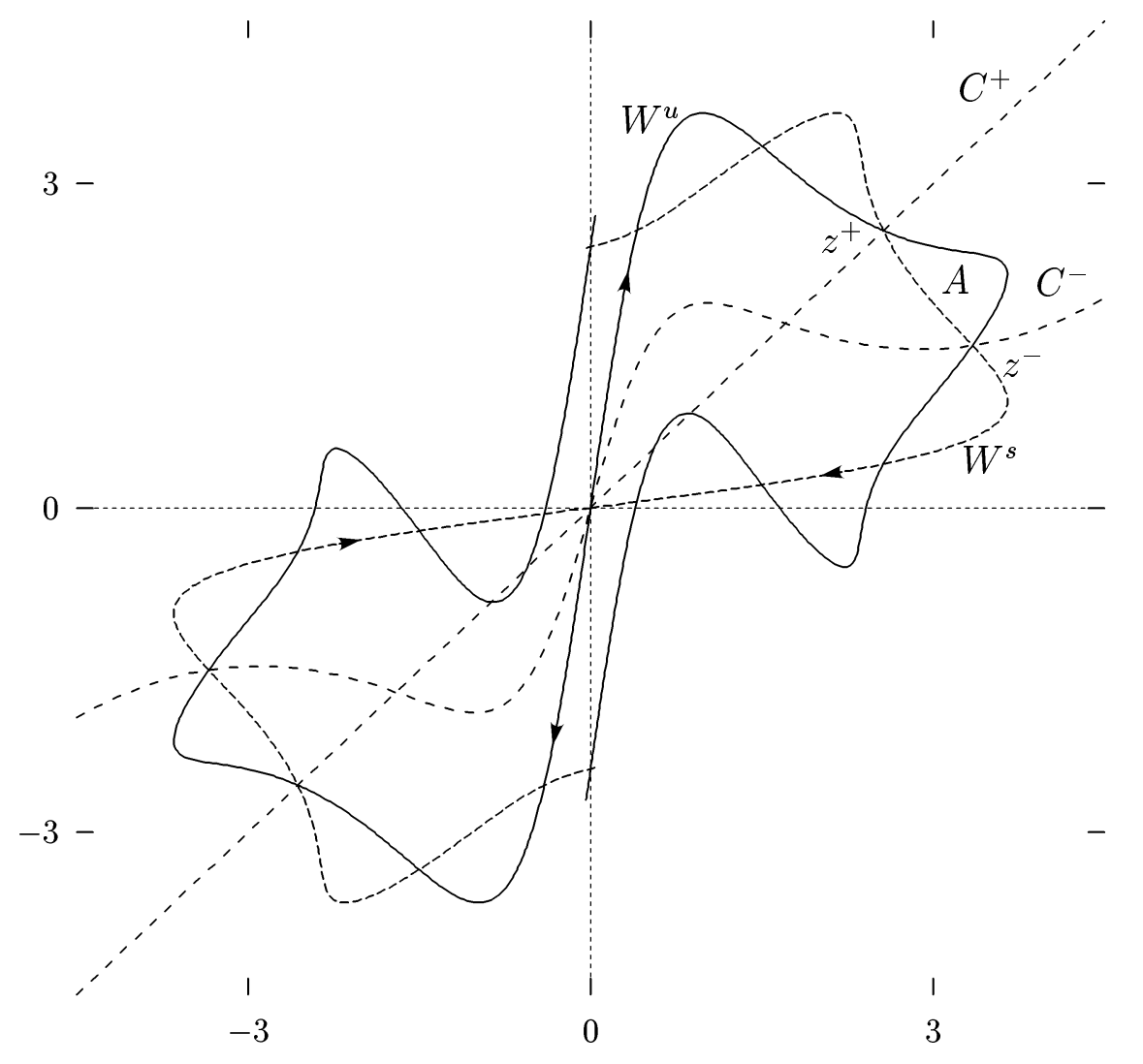

Figure 2: The homoclinic points $z^{ \pm}$and the lobe area $A$ for $\varepsilon V^{\prime}(y)=y^{3} / 40$ and $h=2$.

A natural parameterization is unique except for a translation in the independent variable. To determine it, it is worth looking at the reversors of the map.

Indeed, the involution $R^{+}(x, y):=(y, x)$ is a reversor of the McMillan map $F_{0}$, that is, $F_{0}^{-1}=R^{+} \circ F_{0} \circ R^{+}$. The separatrix $\Lambda$ is $R^{+}$-symmetric, i.e., $R^{+} \Lambda=\Lambda$, and intersects transversely the fixed set $C^{+}:=\left\{z: R^{+} z=z\right\}$ of $R^{+}$in one point $z_{0}^{+}$. The parameterization (2.4) of $\Lambda$ has been chosen to satisfy $z_{0}(0)=z_{0}^{+}$.

Moreover, the involution $R_{0}^{-}:=F_{0} \circ R^{+}$is another reversor of $F_{0}$. The separatrix $\Lambda$ is also $R_{0}^{-}$-symmetric and intersects transversely the fixed set $C_{0}^{-}$of $R_{0}^{-}$in one point $z_{0}^{-}$, and it turns out that $z_{0}(h / 2)=z_{0}^{-}$. The associated orbits $\mathcal{O}_{0}^{+}:=\left\{z_{0}(n h): n \in \mathbb{Z}\right\}$, $\mathcal{O}_{0}^{-}:=\left\{z_{0}(h / 2+n h): n \in \mathbb{Z}\right\}$, are called symmetric homoclinic orbits, since $R^{+} \mathcal{O}_{0}^{+}=$ $\mathcal{O}_{0}^{+}, R_{0}^{-} \mathcal{O}_{0}^{-}=\mathcal{O}_{0}^{-}$.

\section{The perturbed model}

For $\varepsilon \neq 0$, the phase portrait of the exact map (2.2) looks more intricate. The origin is a hyperbolic fixed point with the same characteristic exponent $h$, since the perturbation $\varepsilon U_{1}^{\prime}(y)=\mathrm{O}\left(y^{3}\right)$ does not not contain linear terms at the origin. We denote by $\mathcal{W}^{\mathrm{u}, \mathrm{s}}$ its unstable and stable invariant curves with respect to $F$. Since the map (2.2) is odd, the 
invariant curves are symmetric with respect to the origin, so that we concentrate only on the positive quadrant $\{x, y>0\}$.

By the form of the perturbation, $R^{+}$is also a reversor of $F$, as well as the involution $R^{-}:=F \circ R^{+}$, which is given by $R^{-}(x, y)=\left(x,-y+U^{\prime}(x)\right)$. Their fixed sets $C^{ \pm}=$ $\left\{z: R^{ \pm} z=z\right\}$ are important because $R^{ \pm}\left(\mathcal{W}^{\mathrm{u}}\right)=\mathcal{W}^{\mathrm{s}}$. Consequently, any point in the intersection $C^{ \pm} \cap \mathcal{W}^{\mathrm{u}}$ is a homoclinic point (see figure 2), and gives rise to a symmetric homoclinic orbit.

Since the separatrix $\Lambda$ intersects transversely the unperturbed curve $C_{0}^{ \pm}$at the point $z_{0}^{ \pm}$, there exists a point $z^{ \pm}=z_{0}^{ \pm}+\mathrm{O}(\varepsilon) \in C^{ \pm} \cap \mathcal{W}^{\mathrm{u}}$ and, therefore, there exist at least two symmetric homoclinic orbits on the quadrant $\{x, y>0\}$, for $|\varepsilon|$ small enough. They are called primary since they exist for arbitrary small $|\varepsilon|$.

\section{The regular case}

Along this section, the characteristic exponent $h>0$ will be considered fixed, and then $\varepsilon \rightarrow 0$. In particular, any sentence like "for $|\varepsilon|$ small enough" will mean: "there exists $\varepsilon^{*}(h)>0$ such that for $|\varepsilon|<\varepsilon^{*}(h)$ ". Typically, $\varepsilon^{*}(h)$ will be exponentially small in $h$.

\subsection{The Melnikov theory for exact planar maps}

We now recall some perturbative results to detect the existence of transverse homoclinic orbits for exact maps. For simplicity, we will assume that all the objects are smooth and we shall restrict the discussion to maps on the plane with the usual symplectic structure: the area.

Given the symplectic form $\omega=\mathrm{d} x \wedge \mathrm{d} y=\mathrm{d}(-y \mathrm{~d} x)$ on the plane $\mathbb{R}^{2}$, a map $F: \mathbb{R}^{2} \rightarrow \mathbb{R}^{2}$ is called exact if there exists some function $S: \mathbb{R}^{2} \rightarrow \mathbb{R}$ such that $F^{*}(y \mathrm{~d} x)-y \mathrm{~d} x=\mathrm{d} S$. The function $S$ is called the generating function of $F$ and, except for an additive constant, it is uniquely determined.

Let $F_{0}: \mathbb{R}^{2} \rightarrow \mathbb{R}^{2}$ be an integrable exact diffeomorphism with a separatrix $\Lambda$ to a hyperbolic fixed point $z_{0}^{\infty}$. Next, consider a family of exact diffeomorphisms $F_{\varepsilon}=F_{0}+$ $\varepsilon F_{1}+\mathrm{O}\left(\varepsilon^{2}\right)$, as a general perturbation of the situation above, and let $S_{\varepsilon}=S_{0}+\varepsilon S_{1}+\mathrm{O}\left(\varepsilon^{2}\right)$ be the generating function of $F_{\varepsilon}$.

We introduce the Melnikov potential of the problem as the smooth real-valued function $L: \Lambda \rightarrow \mathbb{R}$ given by

$$
L(z)=\sum_{n \in \mathbb{Z}} \widehat{S}_{1}\left(z_{n}\right), \quad z_{n}=F_{0}{ }^{n}(z), \quad z \in \Lambda,
$$

where $\widehat{S}_{1}: \mathbb{R}^{2} \rightarrow \mathbb{R}$ is defined by $\widehat{S}_{1}=S_{1}-y \mathrm{~d} x\left(F_{0}\right)\left[F_{1}\right]$. (In components, writing $F_{0}=\left(X_{0}, Y_{0}\right), F_{1}=\left(X_{1}, Y_{1}\right), \widehat{S}_{1}$ is simply given by $\widehat{S}_{1}=S_{1}-Y_{0} X_{1}$. $)$ In order to get an absolutely convergent series $(3.1), \widehat{S}_{1}$ is determined by imposing $\widehat{S}_{1}\left(z_{0}^{\infty}\right)=0$.

The differential of $L$ is a geometrical object which gives the $\mathrm{O}(\varepsilon)$-distance between the perturbed invariant curves $\mathcal{W}_{\varepsilon}^{\mathrm{u}, \mathrm{s}}$. More precisely, let $(t, e)$ be some cotangent coordinates adapted to $\Lambda$ - that is, in these coordinates the separatrix $\Lambda$ is given locally by 
$\{e=0\}$ and the symplectic form $\omega$ reads as $\mathrm{d} t \wedge \mathrm{d} e$-and let $\left\{e=E_{\varepsilon}^{\mathrm{u}, \mathrm{s}}(t)\right\}$ be a part of $\mathcal{W}_{\varepsilon}^{\mathrm{u}, \mathrm{s}}$. (Let us recall that cotangent coordinates can be defined in neighborhoods of Lagrangian sub-manifolds [Wei73].) Then, in [DR97b] it is shown that

$$
E_{\varepsilon}^{\mathrm{u}}(t)-E_{\varepsilon}^{\mathrm{s}}(t)=\varepsilon L^{\prime}(t)+\mathrm{O}\left(\varepsilon^{2}\right)
$$

and that the construction above does not depend on the cotangent coordinates used.

The following theorem [DR97b, Theorem 2.1] is a straightforward corollary of this geometric construction.

Theorem 3.1 Under the above notations and hypotheses, the non-degenerate critical point of $L$ are associated to perturbed transverse homoclinic orbits. Moreover, when all the critical points of $L$ are non-degenerate, all the primary homoclinic orbits arising from $\Lambda$ are found in this way. Finally, if $z$ and $z^{\prime}$ are consecutive (in the internal order of the separatrix) non-degenerate critical points of $L$, their associated perturbed homoclinic orbits determine a lobe with area

$$
A=\varepsilon\left[L(z)-L\left(z^{\prime}\right)\right]+\mathrm{O}\left(\varepsilon^{2}\right)
$$

\subsection{The regular analytical result}

We are now ready to apply the theory above to our model. It is worth noting that the knowledge of the natural parameterization (2.4) of the unperturbed separatrix $\Lambda$ will be the crucial point to compute explicitly the Melnikov potential (3.1).

The map $F=F_{0}+\varepsilon F_{1}+\mathrm{O}\left(\varepsilon^{2}\right)$ given in (2.2) is exact with generating function $S(x, y)=-x y+U_{0}(y)+\varepsilon U_{1}(y)$. Writing its expression in components $F_{0}=\left(X_{0}, Y_{0}\right)$, $F_{1}=\left(X_{1}, Y_{1}\right)$, it turns out that $X_{1}=0$, and consequently $\widehat{S}_{1}(x, y)=S_{1}(x, y)=U_{1}(y)$.

The parameterization (2.4) allows us to write the Melnikov potential (3.1) of our problem as

$$
L(t):=L\left(z_{0}(t)\right)=\sum_{n \in \mathbb{Z}} U_{1}\left(y_{0}(t+h n)\right)=\sum_{n \in \mathbb{Z}}[f(t+h n)-g(t+h n)]
$$

where $f(t):=V\left(\xi_{0}(t+h / 2)\right)$ and $g(t):=V_{1} \log \left(1+\xi_{0}(t+h / 2)^{2}\right)$.

We are now confronted to the computation of a series for $L(t)$, which is a doublyperiodic function: $L(t)=L(t+h)=L(t+\pi \mathrm{i})$. Consequently, the explicit computation of $L(t)$ can be performed through the study of its singularities for complex values of the discrete time $t$ [DR96].

For example, $L_{g}(t):=\sum_{n} g(t+h n)$ is easily computed simply by noting that $L_{g}(t)$ has no singularities and, therefore, it must be constant by Liouville's theorem. The exact value of the constant is not important for our purposes, since the intrinsic geometrical object associated to the problem is $L^{\prime}(t)$ rather than $L(t)$.

The computation of $L_{f}(t):=\sum_{n} f(t+h n)$ follows the same lines, although is more complicated. We sketch here the main ideas, and refer to [DR97a] for the details. 
First, we notice that the singularities of $f(t)$ are just located on the set $-h / 2+$ $\pi \mathrm{i} / 2+\pi \mathrm{i} \mathbb{Z}$. Next, we denote by $\sum_{n \in \mathbb{Z}} v_{n}(h) \tau^{2 n}$ the Laurent expansion around $\tau=0$ of the function $\tau \mapsto f(-h / 2+\pi \mathrm{i} / 2-\mathrm{i} h \tau)$, and note that $v_{-n}(h)$ are even entire functions such that $v_{-n}(0)=V_{n}$, for all $n \geq 1$. Finally, we introduce the even entire function

$$
\Theta^{0}(h):=8 \pi \sum_{n \geq 1} \frac{(2 \pi)^{2 n-1}}{(2 n-1) !} v_{-n}(h)=8 \pi \widehat{V}(2 \pi)+\mathrm{O}\left(h^{2}\right),
$$

where $\widehat{V}(\xi):=\sum_{n \geq 1} V_{n} \xi^{2 n-1} /(2 n-1)$ ! is the so-called Borel transform of $V(y)$.

Then, the following asymptotic formula holds for the Melnikov potential $L=L_{f}-$ $L_{g}=L_{f}$ (modulo an additive constant):

$$
L(t)=\mathrm{e}^{-\pi^{2} / h} \cos (2 \pi t / h)\left[-\Theta^{0}(h) / 2+\mathrm{O}\left(\mathrm{e}^{-2 \pi^{2} / h}\right)\right] .
$$

If $V(y)$ is a polynomial, $\Theta^{0}(h)$ can be explicitly computed in a finite number of steps [DR96]. For instance, for the perturbations used in the numerical experiments,

$$
\Theta^{0}(h)=\left\{\begin{array}{ll}
8 \pi^{2} \gamma^{2} h^{-2} & \text { for } V^{\prime}(y)=y \\
\frac{8}{3} \pi^{2} \gamma^{4} h^{-2}\left[1+\pi^{2} h^{-2}\right] & \text { for } V^{\prime}(y)=y^{3}
\end{array} .\right.
$$

From the formula (3.2), it is clear that if $\widehat{V}(2 \pi) \neq 0$ and $h$ is small enough, the set of critical points of the Melnikov potential (3.3) is $h \mathbb{Z} / 2$. All of them are non-degenerate, and parameterize the two unperturbed, symmetric, primary homoclinic orbits $\mathcal{O}_{0}^{ \pm}$. Now, the following result is a corollary of theorem 3.1 .

Theorem 3.2 Assume that $\hat{V}(2 \pi) \neq 0$. Then, for any small enough (but fixed) characteristic exponent $h>0$, there exists a positive constant $\varepsilon^{*}=\varepsilon^{*}(h)$ such that the map (2.2) has exactly two transverse, symmetric, primary homoclinic orbits $\mathcal{O}^{ \pm}$in the quadrant $\{x, y>0\}$, for $0<|\varepsilon|<\varepsilon^{*}$. These orbits determine a lobe with area $A=\varepsilon A_{\mathrm{Mel}}+\mathrm{O}\left(\varepsilon^{2}\right)$, where the first order in $\varepsilon$ approximation $A_{\mathrm{Mel}}$ is given by

$$
A_{\mathrm{Mel}}=L(h / 2)-L(0)=\mathrm{e}^{-\pi^{2} / h}\left[\Theta^{0}(h)+\mathrm{O}\left(\mathrm{e}^{-2 \pi^{2} / h}\right)\right] .
$$

Remark 3.1 We note that $\varepsilon A_{\mathrm{Mel}}$ is the dominant term for the Melnikov formula of the lobe area $A$ only if $|\varepsilon|<\varepsilon^{*}(h)=\mathrm{o}\left(\exp \left(-\pi^{2} / h\right)\right)$. Otherwise, in the case $\varepsilon=\mathrm{O}\left(h^{p}\right)$, the Melnikov theory as described is not useful, since it only gives the very coarse estimate $A=\mathrm{O}\left(h^{2 p}\right)$, and not the desired exponentially small asymptotic behavior.

\section{The singular case}

Along this section, $h \rightarrow 0^{+}$, and we will study analytically and numerically two different situations for the parameter $\varepsilon$ :

- The non-perturbative case: $\varepsilon$ fixed and $h \rightarrow 0^{+}$. 
- The perturbative case: $\varepsilon=\mathrm{o}\left(h^{p}\right)$ and $h \rightarrow 0^{+}$, for some $p \geq 0$.

For the analytical results we only assume that the perturbed potential $V(y)$ is an even entire function. The numerical experiments have been performed for the simplest even perturbed potentials, that is, for the linear perturbation $\varepsilon V^{\prime}(y)=\varepsilon y$, and the cubic one $\varepsilon V^{\prime}(y)=\varepsilon y^{3}$.

\subsection{Singular analytical results}

\section{The non-perturbative case}

The limit $h \rightarrow 0^{+}$in (2.2) is highly singular, since all the interesting dynamics is contained in a $\mathrm{O}(h)$ neighborhood of the origin, which becomes a parabolic point of the map for $h=0$. To see clearly this behavior, we perform the following linear change of variables:

$$
z=C w, \quad C=h\left(\begin{array}{cc}
\lambda^{-1 / 2} & \lambda^{1 / 2} \\
\lambda^{1 / 2} & \lambda^{-1 / 2}
\end{array}\right), \quad z=(x, y), \quad w=(u, v),
$$

that is, we diagonalize the linear part of (2.2) at the origin and we scale by a factor $h$. Then,

$$
\left(C^{-1} \circ F \circ C\right) w=w+h X^{0}(w)+\mathrm{O}\left(h^{2}\right),
$$

where

$$
X^{0}(u, v)=\left(u-\eta(u+v)^{3},-v+\eta(u+v)^{3}\right), \quad \eta=1-\left(V_{1}+2 V_{2}\right) \varepsilon,
$$

is a Hamiltonian vector field, with associated Hamiltonian

$$
H^{0}(u, v)=u v-\eta(u+v)^{4} / 4 .
$$

Expression (4.1) shows clearly that $F$ is $\mathrm{O}(h)$-close to the identity, and that, after the change of variables $z=C w$, the map (2.2) asymptotes to the Hamiltonian flow associated to the vector field (4.2) when $h \rightarrow 0^{+}$. When such situation takes place, it is known [Fon89] that the map (2.2) will have homoclinic points to the origin for any small enough $h$, if and only if the limit Hamiltonian flow has a homoclinic orbit to the origin.

From the expression (4.3), we see that the zero level $\left\{H^{0}(u, v)=0\right\}$ contains homoclinic connections to the origin if and only if $\eta>0$, i.e., if

$$
\left(V_{1}+2 V_{2}\right) \varepsilon<1 \text {. }
$$

Assuming $\eta>0$, the homoclinic orbit of the Hamiltonian (4.3) is given by

$$
w^{0}(t)=\eta^{-1 / 2}\left(\frac{\cosh t-\sinh t}{2 \cosh ^{2} t}, \frac{\cosh t+\sinh t}{2 \cosh ^{2} t}\right),
$$

which is analytic on the strip $\{t \in \mathbb{C}:|\Im t|<d:=\pi / 2\}$. In this situation, it is also well-known [FS90] that the splitting size is $\mathrm{O}(\exp (-\beta / h))$, for all $\beta<2 \pi d=\pi^{2}$. We summarize these first analytical results. 
Theorem 4.1 For any real $\varepsilon$ verifying (4.4), and any $\beta \in\left(0, \pi^{2}\right)$, there exists $N=$ $N(\varepsilon, \beta)>0$ such that the area of the lobe between the invariant curves of the map (2.2) satisfies:

$$
|A| \leq N \mathrm{e}^{-\beta / h} \quad\left(\varepsilon \text { fixed, } h \rightarrow 0^{+}\right)
$$

\section{The perturbative case}

The previous theorem gives only an upper bound for the lobe area and not an asymptotic one (the constant $N(\varepsilon, \beta)$ can blow up when $\beta \rightarrow \pi^{2}$ ). In particular, it does not exclude the case $A=0$, that is, it cannot detect effective splitting of separatrices. In the perturbative case $\varepsilon=\mathrm{o}\left(h^{p}\right)$, for $p>6$, the following theorem gives an asymptotic expression for the lobe area in terms of the Melnikov potential, and establishes transversal splitting of separatrices. The version presented here is slightly more general that the one contained in [DR97a], since we have dropped out the hypothesis $V^{\prime}(0)=2 V_{1}=0$ of that paper.

Theorem 4.2 Assume that $\varepsilon=\mathrm{o}\left(h^{p}\right), p>6$. Then, if $\hat{V}(2 \pi) \neq 0$, there exists $h^{*}>0$ such that the map (2.2) has exactly two transverse, symmetric, primary homoclinic orbits in the first quadrant, for all $0<h<h^{*}$. Moreover, they enclose a lobe with area

$$
A=\varepsilon \mathrm{e}^{-\pi^{2} / h}\left[8 \pi \widehat{V}(2 \pi)+\mathrm{O}\left(h^{2}\right)\right] \quad\left(h \rightarrow 0^{+}\right) .
$$

If $\widehat{V}(2 \pi)=0$, there may exist more primary homoclinic orbits, but the area of any lobe is $\mathrm{O}\left(\varepsilon h^{2} \mathrm{e}^{-\pi^{2} / h}\right)$.

Proof. For $V_{1}=0$, the result above is just the Main Theorem of [DR97a]. For $V_{1} \neq 0$, the perturbative potential $U_{1}(y)=V(y)-V_{1} \log \left(1+y^{2}\right)$ in (2.2) is not longer an entire function, due to the term $g(y):=V_{1} \log \left(1+y^{2}\right)$, and the Main Theorem of [DR97a] cannot be applied directly.

However, this result follows from the following observations:

1. As we have already seen in section 3.2 , the Melnikov potential $L(t)$ is not affected by the contribution of $L_{g}(t)$.

2. One can easily bound $g^{\prime}\left(\xi^{0}(t)+\eta\right)$ in such a way that the estimates of Lemma 3.5 in [DR97a] do not change.

Now, the rest of arguments in [DR97a] remain applicable, and the result follows.

We finish this account of analytical results by remarking that, to the best of our knowledge, the result above, jointly with [DR97a], are the first analytical results about asymptotics for singular separatrix splitting for a map with a complete and rigorous proof. 


\subsection{Singular numerical results}

In the regular case, we dispose of formula (3.5) for the lobe area $A$, in terms of an even analytic function $\Theta^{0}(h)$, with a fairly simple expression (3.4) for $V^{\prime}(y)=y, y^{3}$.

These regular results suggest that in the singular case, for every fixed $\varepsilon$ verifying (4.4), the actual formula for the lobe area may have the form

$$
A=\varepsilon \mathrm{e}^{-\pi^{2} / h}\left[\Theta^{\varepsilon}(h)+\mathrm{O}\left(\mathrm{e}^{-2 \pi^{2} / h}\right)\right], \quad\left(\text { fixed } \varepsilon, h \rightarrow 0^{+}\right),
$$

for a function $\Theta^{\varepsilon}(h)$ given by an asymptotic series of the form

$$
\Theta^{\varepsilon}(h) \sim \sum_{n \geq 0} \Theta_{n}^{\varepsilon} h^{2 n}, \quad\left(\text { fixed } \varepsilon, h \rightarrow 0^{+}\right) .
$$

The sign $\sim$ means that the series $\sum_{n>0} \Theta_{n}^{\varepsilon} h^{2 n}$ needs not to be convergent, but only asymptotic, that is, if one retains a finite number of the first successive terms, the error has the order of the first missing term:

$$
\left|\Theta^{\varepsilon}(h)-\sum_{n=0}^{N} \Theta_{n}^{\varepsilon} h^{2 n}\right|=\mathrm{O}\left(h^{2 N+2}\right) .
$$

We are interested in computing a relevant number of the coefficients $\Theta_{n}^{\varepsilon}$ for some significant perturbations $\varepsilon V^{\prime}(y)$, in such a way that we can measure their asymptotic behavior, and describe the analytical properties of the function $\Theta^{\varepsilon}(h)$.

To such end, once we have chosen a perturbation $\varepsilon V^{\prime}(y)$, we compute the lobe area $A$ with a relative error less than $\rho$, for a net $\mathcal{N}$ of values of the characteristic exponent $h$. We take an equidistant net in $h^{2}$, due to the fact that we expect that the asymptotic series (4.6) will contain only even powers of $h$. That is, we take

$$
\mathcal{N}=\left\{h_{j}:=j^{1 / 2} \delta: j=1, \ldots, \ell+1\right\}
$$

for some (relatively) small positive number $\delta$ and some (relatively) large natural $\ell$.

We have chosen the values

$$
\rho=10^{-900}, \quad \delta=0.001, \quad \ell=99 .
$$

Another choices are also possible, but, taking into account our purposes, it is not worth taking values of $\rho$ much smaller than $\exp \left(-2 \pi^{2} / h_{\ell+1}\right)$. Let us explain this.

We do not know how to compute directly the function $\Theta^{\varepsilon}(h)$, but only how to approximate it by $\varepsilon^{-1} \exp \left(\pi^{2} / h\right) A$. Once obtained the approximated values of $\Theta^{\varepsilon}(h)$ on the net $\mathcal{N}$, they will be the input of some algorithm which computes the first $\ell+1$ asymptotic coefficients $\Theta_{n}^{\varepsilon}$. This explains why it is rather absurd to take $\rho$ too much small, being $\rho \approx \exp \left(-2 \pi^{2} / h\right)$ the greatest accuracy we can expect on approximating $\Theta^{\varepsilon}(h)$ by $\varepsilon^{-1} \exp \left(\pi^{2} / h\right) A$. Since all the values in the net are computed with the same accuracy, we must take $\rho$ not much smaller than $\exp \left(-2 \pi^{2} / h_{\ell+1}\right)=\max _{1 \leq j \leq \ell+1} \exp \left(-2 \pi^{2} / h_{j}\right)$. 



Figure 3: $\Xi_{n}^{\varepsilon}$ vs. $n$, for $\varepsilon=0.1$. The dashed lines correspond to the limit value $\Xi_{\infty}^{\varepsilon}$, which is found by extrapolation. Left: $V^{\prime}(y)=y$ and $\Xi_{\infty}^{\varepsilon}=-9.7737740885 \ldots \times 10^{-3}$. Right: $V^{\prime}(y)=y^{3}$ and $\Xi_{\infty}^{\varepsilon}=-4.6302913918 \ldots \times 10^{-1}$.

An interpolation method based on Neville's algorithm has been used to compute the asymptotic coefficients of $\Theta^{\varepsilon}(h)$ from the values on the net $\mathcal{N}$. That is, we compute the polynomial $P^{\varepsilon}(h)=\sum_{n=0}^{\ell} P_{n}^{\varepsilon} h^{2 n}$ which interpolates $\Theta^{\varepsilon}(h)$ on $\mathcal{N}$, and next we approximate $\Theta_{n}^{\varepsilon}$ by $P_{n}^{\varepsilon}$, for $n=0, \ldots, \ell$. Although equidistant interpolation using polynomials of high degree (in our case, degree $\ell$ in $h^{2}$ ) is in some cases an ill-conditioned problem, we have checked that the coefficients $\Theta_{n}^{\varepsilon}$ so obtained are accurate enough for our purposes. Concretely, with the choice (4.7), this method gives at least $860-9 n$ significant decimals digits for $\Theta_{n}^{\varepsilon}, n=0, \ldots, 95$. (The accuracy decreases as $n$ increases, but this seems unavoidable.) This can been checked simply by studying the dependence of the coefficients $\Theta_{n}^{\varepsilon}$ on the precision $\rho$ and the degree $\ell$.

\section{The non-perturbative case}

To avoid the factorial increase of the coefficients $\Theta_{n}^{\varepsilon}$ that is observed empirically, we introduce other coefficients $\Xi_{n}^{\varepsilon}$ defined by

$$
\Theta_{n}^{\varepsilon}=(2 n) !\left(2 \pi^{2}\right)^{-2 n}(2 n)^{4} \Xi_{n}^{\varepsilon},
$$

expecting that the coefficients $\Xi_{n}^{\varepsilon}$ will tend to a certain constant $\Xi_{\infty}^{\varepsilon}$, as $n \rightarrow \infty$. Figure 3 shows clearly this behavior for the two different perturbations: the linear case $V^{\prime}(y)=y$, and the cubic case $V^{\prime}(y)=y^{3}$. The limit constants $\Xi_{\infty}^{\varepsilon}$ are found by applying an extrapolation method on the coefficients $\Xi_{n}^{\varepsilon}$ (see also the table 1 ).

In particular, we have that $\left|\Theta_{n}^{\varepsilon}\right| \leq C \rho^{2 n} \Gamma(2 n+5)$ for some constant $C$ and $\rho=1 / 2 \pi^{2}$, that is, the function $\Theta^{\varepsilon}(h)$ of (4.6) is Gevrey-1 of type $\rho=1 / 2 \pi^{2}$ with respect to the variable $h$.

We now summarize these numerical results.

Numerical result 4.1 For the linear and cubic perturbations, the following asymptotic 
expansion for the lobe area $A$ holds

$$
A \sim \varepsilon \mathrm{e}^{-\pi^{2} / h} \sum_{n \geq 0} \Theta_{n}^{\varepsilon} h^{2 n} \quad\left(\varepsilon \text { fixed, } h \rightarrow 0^{+}\right),
$$

where the coefficients $\Theta_{n}^{\varepsilon}$ verify

$$
\Theta_{n}^{\varepsilon}=(2 n) !\left(2 \pi^{2}\right)^{-2 n}(2 n)^{4}\left[\Xi_{\infty}^{\varepsilon}+\mathrm{O}\left(n^{-1}\right)\right],
$$

as $n \rightarrow+\infty$, for some constant $\Xi_{\infty}^{\varepsilon} \neq 0 .\left(\Xi_{\infty}^{\varepsilon}<0\right.$ for $\varepsilon>0$.)

In other words, the formula (4.5) for the lobe area holds for an even $\Theta^{\varepsilon}(h)$ such that its Borel transform $\widehat{\Theta^{\varepsilon}}(h)=\sum_{n} \Theta_{n}^{\varepsilon} \xi^{2 n-1} /(2 n-1)$ ! is convergent for $|\xi|<2 \pi^{2}$.

Of course, we believe that the numerical result above holds for any even entire perturbative potential $\varepsilon V(y)$.

\section{The perturbative case}

We now check that all the previous objects $\Theta^{\varepsilon}(h), \Theta_{n}^{\varepsilon}, \Xi_{\infty}^{\varepsilon}$, tend to well-defined limits, as $\varepsilon \rightarrow 0$.

We begin by describing the results connecting the Gevrey-1 function $\Theta^{\varepsilon}(h)$ with the Melnikov prediction $\Theta^{0}(h)$ given in (3.2). Applying formula (3.4), we immediately get that

$$
\Theta^{0}(h)=8 \pi^{2} \gamma^{2} h^{-2}=8 \pi^{2}\left[1+\frac{h^{2}}{3}+\frac{16}{45} h^{4}+\frac{8}{315} h^{6}+\mathrm{O}\left(h^{8}\right)\right],
$$

for the linear perturbation $\varepsilon V^{\prime}(y)=\varepsilon y$, and

$$
\begin{aligned}
\Theta^{0}(h) & =\frac{8}{3} \pi^{2} \gamma^{4} h^{-2}\left[1+\pi^{2} h^{-2}\right] \\
& =\frac{8}{3} \pi^{4}\left[1+\left(1+\frac{2}{3} \pi^{2}\right) h^{2}+\left(\frac{2}{3}+\frac{1}{5} \pi^{2}\right) h^{4}+\left(\frac{1}{5}+\frac{34}{945} \pi^{2}\right) h^{6}+\mathrm{O}\left(h^{8}\right)\right],
\end{aligned}
$$

for the cubic one $\varepsilon V^{\prime}(y)=\varepsilon y^{3}$.

The numerical results comparing $\Theta^{\varepsilon}(h)$ with $\Theta^{0}(h)$, for $\varepsilon \rightarrow 0$, are displayed in figure 4. On the left-hand side of figure 4 it is shown that $\Theta^{\varepsilon}(h)$ tends uniformly to $\Theta^{0}(h)$ as $\varepsilon \rightarrow 0$, whereas on the right-hand side we have checked that $\varepsilon^{-1}\left[\Theta^{\varepsilon}(h)-\Theta^{0}(h)\right]$ tends uniformly to some continuous function. Thus, we conclude that

$$
\Theta^{\varepsilon}(h)=\Theta^{0}(h)+\mathrm{O}(\varepsilon), \quad \text { uniformly for } h \in(0,1] .
$$

Next, we compare the coefficients $\Theta_{n}^{\varepsilon}$ in the expression (4.6) of the function $\Theta^{\varepsilon}(h)$ with the coefficients $\Theta_{n}^{0}$ in the Taylor expansion of $\Theta^{0}(h)$, as $\varepsilon \rightarrow 0$.

The results about the convergence of some of the coefficients are shown in figure 5 , where one can see that $\Theta_{n}^{\varepsilon}$ tends to $\Theta_{n}^{0}$ as $\varepsilon \rightarrow 0$. It is worth noting that we cannot expect any kind of uniform convergence in $n \geq 0$, since $\Theta^{\varepsilon}(h)$ is a Gevrey- 1 function (in particular, divergent), whereas $\Theta^{0}(h)$ is an entire function. 



Figure 4: Up \& Left: graphs of $h \mapsto \Theta^{\varepsilon}(h)$, for $V^{\prime}(y)=y$ and $\varepsilon=0,0.01,0.03,0.05,0.07$, from top to bottom. Up \& Right: graphs of $h \mapsto\left(\Theta^{\varepsilon}(h)-\Theta^{0}(h)\right) / \varepsilon$, for $V^{\prime}(y)=y$ and $\varepsilon=10^{-5}, 10^{-4}, 10^{-3}, 10^{-2}$, from top to bottom. Down \& Left: graphs of $h \mapsto \Theta^{\varepsilon}(h)$, for $V^{\prime}(y)=y^{3}$ and $\varepsilon=0,0.01,0.03,0.05,0.07$, from top to bottom. Down \& Right: graphs of $h \mapsto\left(\Theta^{\varepsilon}(h)-\Theta^{0}(h)\right) / \varepsilon$, for $V^{\prime}(y)=y^{3}$ and $\varepsilon=0.04,0.02,0.01,0.001$, from top to bottom.
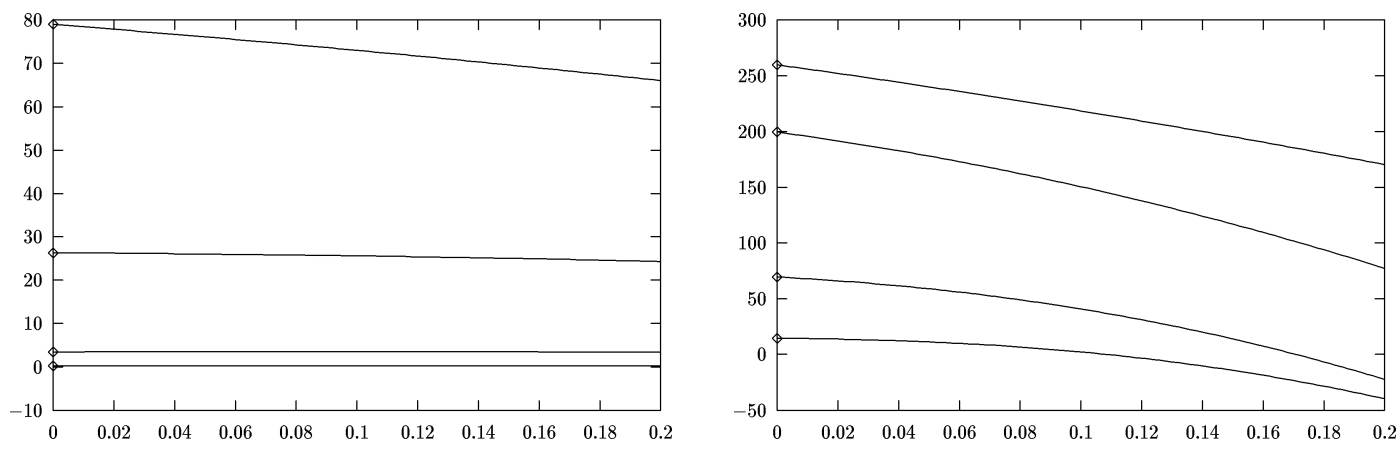

Figure 5: $\Theta_{n}^{\varepsilon}$ vs. $\varepsilon$, for $n=0,1,2,3$, from top to bottom. The marked points correspond to the values $\Theta_{n}^{0}$-that is, $\varepsilon=0$-, obtained by the Melnikov approach. Left: $V^{\prime}(y)=y$, where $\Theta_{0}^{0}=8 \pi^{2}, \Theta_{1}^{0}=8 \pi^{2} / 3, \Theta_{2}^{0}=16 \pi^{2} / 45$, and $\Theta_{3}^{0}=8 \pi^{2} / 315$. Right: $V^{\prime}(y)=y^{3}$, where $\Theta_{0}^{0}=8 \pi^{4} / 3, \Theta_{1}^{0}=8 \pi^{2}\left(3+2 \pi^{2}\right) / 9, \Theta_{2}^{0}=8 \pi^{2}\left(10+3 \pi^{2}\right) / 45$, and $\Theta_{3}^{0}=8 \pi^{2}(189+$ $\left.34 \pi^{2}\right) / 2835$. 
Table 1: Computed values of $\Xi_{\infty}^{\varepsilon}$ for the linear and cubic perturbations. The last row contains the values of $\Xi_{\infty}^{0}=\lim _{\varepsilon \rightarrow 0} \varepsilon^{-1} \Xi_{\infty}^{\varepsilon}$ found by extrapolation.

\begin{tabular}{c|c|c}
$\varepsilon$ & \multicolumn{2}{|c}{$\varepsilon^{-1} \Xi_{\infty}^{\varepsilon}$} \\
\hline & $V^{\prime}(y)=y$ & $V^{\prime}(y)=y^{3}$ \\
\hline $10^{-1}$ & $-0.09773774088 \ldots$ & $-4.6302913918 \ldots$ \\
$10^{-2}$ & $-0.12084203100 \ldots$ & $-5.2302522778 \ldots$ \\
$10^{-3}$ & $-0.12295874638 \ldots$ & $-5.3224971013 \ldots$ \\
$10^{-4}$ & $-0.12316850220 \ldots$ & $-5.3322442111 \ldots$ \\
$10^{-5}$ & $-0.12318945876 \ldots$ & $-5.3332243659 \ldots$ \\
$10^{-6}$ & $-0.12319155423 \ldots$ & $-5.3333224360 \ldots$ \\
\hline$\rightarrow 0$ & $-0.12319178706 \ldots$ & $-5.3333333333 \ldots$
\end{tabular}

Finally, we study the behavior of the limit constant $\Xi_{\infty}^{\varepsilon}$ that appears in the Numerical Result 4.1, for $\varepsilon \rightarrow 0$.

We give in table 1 the values of $\varepsilon^{-1} \Xi_{\infty}^{\varepsilon}$ for several values of the perturbation strength $\varepsilon$. It is evident from this table that $\varepsilon^{-1} \Xi_{\infty}^{\varepsilon}=\Xi_{\infty}^{0}+\mathrm{O}(\varepsilon)$, for some constant $\Xi_{\infty}^{0}=-12 \pi^{-4}$ for the linear perturbation, and $\Xi_{\infty}^{0}=-16 / 3$ for the cubic one.

We summarize now the numerical results found for the perturbative case.

Numerical result 4.2 For the linear and cubic perturbations, the objects $\Theta^{\varepsilon}(h), \Theta_{n}^{\varepsilon}$, $\Xi_{\infty}^{\varepsilon}$, introduced in the Numerical Result 4.1, tend to well-defined limits, as $\varepsilon \rightarrow 0$. More precisely,

1. $\Theta^{\varepsilon}(h)=\Theta^{0}(h)+\mathrm{O}(\varepsilon)$, uniformly in $h \in(0,1]$.

2. $\Theta_{n}^{\varepsilon}=\Theta_{n}^{0}+\mathrm{O}(\varepsilon)$, non-uniformly in $n \geq 0$.

3. $\Xi_{\infty}^{\varepsilon}=\varepsilon \Xi_{\infty}^{0}+\mathrm{O}\left(\varepsilon^{2}\right)$, where $\Xi_{\infty}^{0}=\left\{\begin{array}{ll}-12 \pi^{-4} & \text { if } V^{\prime}(y)=y \\ -16 / 3 & \text { if } V^{\prime}(y)=y^{3}\end{array}\right.$.

Again, we believe that the numerical results above hold for any even entire perturbative potential $\varepsilon V(y)$. Concerning the value of $\Xi_{\infty}^{0}$, we conjecture that

$$
V(y) \in \mathbb{Q}[y] \Rightarrow \Xi_{\infty}^{0} \in \mathbb{Q}[\pi] .
$$

\section{The computations}

In this section, we will express the lobe area as a difference of homoclinic actions. We also explain how to compute this exponentially small difference with an arbitrary accuracy as fast as possible. 


\subsection{MacKay-Meiss-Percival action principle}

Let $F$ be an exact map on the plane with the usual symplectic structure $\omega=\mathrm{d} x \wedge \mathrm{d} y$ and let $S$ be its generating function: $F^{*} y \mathrm{~d} x-y \mathrm{~d} x=\mathrm{d} S$. Assume the $z_{\infty}$ is a hyperbolic fixed point of $F$ and let $\mathcal{W}^{\mathrm{u}, \mathrm{s}}$ be its associated unstable and stable invariant curves. Given a homoclinic orbit $\mathcal{O}=\left(z_{n}\right)_{n \in \mathbb{Z}}$ of $F$-that is, $\mathcal{O} \subset\left(\mathcal{W}^{\mathrm{u}} \cap \mathcal{W}^{\mathrm{s}}\right) \backslash\left\{z_{\infty}\right\}$ and $F\left(z_{n}\right)=z_{n+1}$-we define its homoclinic action as

$$
W[\mathcal{O}]=\sum_{n \in \mathbb{Z}} S\left(z_{n}\right)
$$

where, in order to get an absolutely convergent series, the generating function $S$ has been determined by imposing $S\left(z_{\infty}\right)=0$. Given an integer $N$, we denote by $\eta^{\mathrm{u}, \mathrm{s}}(N)$ the paths contained in the invariant curves $\mathcal{W}^{\mathrm{u}, \mathrm{s}}$ from the hyperbolic point $z_{\infty}$ to the homoclinic one $z_{N}$. Then, the following formulae hold [MMP84, Eas91, DR97b]

$$
\sum_{n<N} S\left(z_{n}\right)=\int_{\eta^{\mathrm{u}}(N)} y \mathrm{~d} x, \quad \sum_{n \geq N} S\left(z_{n}\right)=\int_{\eta^{\mathrm{s}}(N)} y \mathrm{~d} x .
$$

These formulae are the key tool to get a computable expression for the lobe area $A$. Let $z^{ \pm}$be two homoclinic points such that the pieces of the invariant curves between them do not contain other points of their orbits. These pieces enclose a region called lobe. Let $\mathcal{O}^{ \pm}$be the homoclinic orbits generated by $z^{ \pm}$, and set $\eta=\eta^{\mathrm{u}}-\eta^{\mathrm{s}}$, where $\eta^{\mathrm{u}, \mathrm{s}} \subset \mathcal{W}^{\mathrm{u}, \mathrm{s}}$ are paths from $z^{+}$to $z^{-}$. Thus, $A=\oint_{\eta} y \mathrm{~d} x$ is the algebraic area of the lobe; the sign of $A$ depends on the way the perturbed curves cross: $A>0$ if and only if $\eta$ is traveled clockwise, like in figure 2. Finally, from equations (5.1) and (5.2), the lobe area $A$ can be expressed as a difference of homoclinic actions:

$$
A=W\left[\mathcal{O}^{-}\right]-W\left[\mathcal{O}^{+}\right]
$$

\subsection{Multiple-precision arithmetic}

To motivate the multiple-precision arithmetic used in the computations, we note that the lobe areas we want compute are $\mathrm{O}\left(\exp \left(-\pi^{2} / h\right)\right)$, whereas the homoclinic actions $W\left[\mathcal{O}^{ \pm}\right]$are much larger since they are of the same order as the region enclosed by the unperturbed separatrix, which is $\mathrm{O}\left(h^{3}\right)$. Thus, equation (5.3) must be carefully used due to the strong cancellation in the difference $W\left[\mathcal{O}^{-}\right]-W\left[\mathcal{O}^{+}\right]$. Even for moderate values $h$, this causes an important loss of significant digits, which can only be overcome computing the actions with more correct digits than the lost ones. For instance, setting $h=0.1$, numerical computations with $\varepsilon V^{\prime}(y)=y^{3} / 10$ give

$$
W\left[\mathcal{O}^{+}\right] \simeq 7.02677 \times 10^{-4} \simeq W\left[\mathcal{O}^{-}\right], \quad A \simeq 3.01433 \times 10^{-42},
$$

so that in order to get at least one correct (decimal) digit for the lobe area $A$ one must have approximately 40 correct digits for the actions $W\left[\mathcal{O}^{ \pm}\right]$. This exceeds the range of a quadruple-precision. 
The number $P$ of decimal digits used in the computations is determined by the following formula:

$$
P=Q+\left[\pi^{2} h^{-1} \log _{10} \mathrm{e}\right]+20,
$$

where $Q$ is the number of significant decimal digits required for the lobe area (usually $Q=100$ or $Q=900$ ), and [.] stands for integer part. The second term is a good approximation for the decimal digits lost by cancellation, and the last one is a security term.

The multiple-precision routines have been performed following the algorithms contained in the Knuth's book [Knu69]. We have avoided the use of external packages in order to have a total control on the program.

The use of an expensive multiple-precision arithmetic encourages us to study maps as "cheap" as possible. Accordingly, we have restricted the experiments to the linear $\left(V^{\prime}(y)=y\right)$ and cubic $\left(V^{\prime}(y)=y^{3}\right)$ cases. For numerical purposes, the representation (2.1) is the one that involves less operations. Given $\varepsilon$ and $h>0$, one computes $\mu=\cosh h, \mu_{0}=\mu-\varepsilon V_{1}$, and then, in the linear case, each evaluation of (2.1) requires one division, two products, and three sums. In the cubic case, just one more product is needed.

\subsection{Invariant curves}

Local invariant curves associated to weakly hyperbolic fixed points must be developed up to high order (see [Sim90] for general comments). This fact is crucial to get the lobe area with the required accuracy as fast as possible: the initial iterates can then be taken far enough from the hyperbolic fixed point and the homoclinic points $z^{ \pm}$can be attained in a few iterations. In this way, undesirable accumulation of rounding errors due to the large amount of operations is avoided and computing time is reduced.

It is very well-known that there exist analytic parameterizations $\sigma^{\mathrm{u}, \mathrm{s}}: \mathbb{R} \rightarrow \mathcal{W}^{\mathrm{u}, \mathrm{s}}$ of the invariant curves such that $F\left(\sigma^{\mathrm{u}}(r)\right)=\sigma^{\mathrm{u}}(\lambda r)$ and $F\left(\sigma^{\mathrm{s}}(r)\right)=\sigma^{\mathrm{s}}\left(\lambda^{-1} r\right)$, where $\lambda$ is the characteristic multiplier of the hyperbolic point. Such parameterizations conjugate the map $F$ to $r \rightarrow \lambda^{ \pm} r$ on the invariant curves, and are determined except for a multiplicative constant in the variable $r$. (A natural parameterization is obtained via the change of variables $r=\exp t$.)

In order to accelerate the numerical computation of these parameterizations we must take advantage of the symmetries, reversors, and peculiarities of the map (2.2).

First, $\sigma^{\mathrm{u}, \mathrm{s}}$ are odd, since so is $F$. Second, the reversors $R^{+}, R^{-}=F \circ R^{+}$, allow us to obtain a parameterization of the stable curve in terms of the unstable one:

$$
\sigma^{\mathrm{s}}(r):=R^{+}\left(\sigma^{\mathrm{u}}(r)\right)=R^{-}\left(\sigma^{\mathrm{u}}(\lambda r)\right) .
$$

Finally, the particular form of the map (2.2) implies that $\sigma^{\mathrm{u}}(r)$ can be written as

$$
\sigma^{\mathrm{u}}(r)=\left(\zeta\left(\lambda^{-1 / 2} r\right), \zeta\left(\lambda^{1 / 2} r\right)\right)
$$


for some analytic odd function $\zeta: \mathbb{R} \rightarrow \mathbb{R}$ such that

$$
\zeta(\lambda r)+\zeta\left(\lambda^{-1} r\right)=U^{\prime}(\zeta(r)) .
$$

Therefore, to get the Taylor expansion of the invariant curves it is enough to solve equation (5.6). Set $\zeta(r)=\sum_{k \geq 0} \zeta_{k} r^{2 k+1}$ and $Q(\zeta(r))=\sum_{k \geq 0} q_{k} r^{2 k+1}$, where $Q(y):=$ $U^{\prime}(y)-2 \mu y=\mathrm{O}\left(y^{3}\right)$. From (5.6), we get $\left[\lambda^{2 k+1}-2 \mu+\lambda^{-(2 k+1)}\right] \zeta_{k}=q_{k}$, for all $k \geq 0$. Since $Q(y)$ begins with cubic terms, $q_{0}$ is zero and $q_{k}$ only depends on $\zeta_{0}, \ldots, \zeta_{k-1}$. Besides, $2 \mu=\lambda+\lambda^{-1}$ (see equalities (2.3)) implies that $\lambda^{\ell}-2 \mu+\lambda^{-\ell}=0$ if and only if $\ell= \pm 1$. Thus, the coefficient $\zeta_{0}$ is the free parameter that multiplies the variable $r$, and

$$
\zeta_{k}=\left[\lambda^{2 k+1}-2 \mu+\lambda^{-(2 k+1)}\right]^{-1} q_{k}, \quad k \geq 1 .
$$

If all the coefficients are known up to the index $k-1$, we can compute successively $q_{k}$ and $\zeta_{k}$, and this recurrent process allows us to compute the coefficients $\zeta_{k}$ up to any fixed index $K$.

To choose adequately $\zeta_{0}$, we take into account that in the unperturbed case $\varepsilon=0$, the parameterization $\zeta_{0}(r)$ is given by $\zeta_{0}(\exp t)=\xi_{0}(t)$ (see (2.4) and (2.5)), and it takes the form

$$
\zeta_{0}(r)=2 \gamma \frac{r}{1+r^{2}}=2 \gamma \sum_{k \geq 0}(-1)^{k} r^{2 k+1},
$$

i.e., $\zeta_{0}(r)$ only has odd Taylor coefficients given by $(-1)^{k} 2 \gamma$.

In the perturbed case, we choose $\zeta_{0}=2 \gamma$ to get a controlled growth for the coefficients $\zeta_{k}$ :

$$
\zeta_{k} \approx(-1)^{k} \zeta_{0}=(-1)^{k} 2 \gamma
$$

This stable behavior of the coefficients $\zeta_{k}$ is particularly suitable for their numerical computation, and makes the previous algorithm very robust in avoiding cancellation problems.

\subsection{Homoclinic points}

In order to find numerically the symmetric homoclinic points $z^{ \pm} \in C^{ \pm}$, we move along the unstable curve $\mathcal{W}^{\mathrm{u}}$ to the first point that intersects $C^{ \pm}$. We explain the process for $z^{+}$; the computation of $z^{-}$follows the same lines.

First, given the number $P$ of decimal digits used in the arithmetic, and an order $K$ for the invariant curve expansions, we must choose a positive number $\delta$ such that

$$
E_{K}(\delta):=\left|\zeta(\delta)-\sum_{k \leq K} \zeta_{k} \delta^{2 k+1}\right|=\left|\sum_{k>K} \zeta_{k} \delta^{2 k+1}\right|<\rho:=10^{-P},
$$

and as large as possible, because the size of $\delta$ determines the number $N$ of iterates needed to reach the homoclinic point. From equation (5.8), we get that

$$
E_{K}(\delta)<2 \gamma \delta^{2 K+3}<\delta^{2 K+3},
$$


for $h$ small and $\delta \in(0,1)$. Thus, a good choice is $\delta^{2 K+3}=\rho=10^{-P}$, that is,

$$
\delta=10^{-P /(2 K+3)} .
$$

Once we have determined $\delta$, we find the first natural $N$ such that $F^{N}\left(\sigma^{\mathrm{u}}(\delta)\right)$ and $F^{N+1}\left(\sigma^{\mathrm{u}}(\delta)\right)=F^{N}\left(\sigma^{\mathrm{u}}(\lambda \delta)\right)$ are separated by $C^{+}=\{y=x\}$, so that the function

$$
g^{+}(r)=\pi_{1} F^{N}\left(\sigma^{\mathrm{u}}(r)\right)-\pi_{2} F^{N}\left(\sigma^{\mathrm{u}}(r)\right),
$$

has a zero $\hat{r}^{+}$in the interval $[\delta, \lambda \delta]$. Here, $\pi_{1}(z)$ and $\pi_{2}(z)$ stand for the projections over the first and second components of $z$, respectively.

Next, we use Newton's method to determine $\hat{r}^{+}$with the precision $\rho$ we are dealing with. For the sake of efficiency, we first work in double-precision and the result is later refined by doubling the number of digits in the multiple-precision arithmetic after each Newton's iteration. (The convergence of Newton method is quadratic.) In this way, the complete Newton's method takes (at most) thrice what the last Newton's iteration takes.

Finally, $z^{+}=F^{N}\left(\sigma^{\mathrm{u}}\left(\hat{r}^{+}\right)\right)=\sigma^{\mathrm{u}}\left(r^{+}\right)$is the homoclinic point over $C^{+}$we are looking for, where $r^{+}=\lambda^{N} \hat{r}^{+}$. In the unperturbed case $r^{+}=1$, because $\zeta_{0}(r)=\zeta_{0}(1 / r)$, see (5.7). Therefore, for moderate perturbation strengths $\varepsilon$,

$$
\mathrm{e}^{-N h}=\lambda^{-N} \approx \hat{r}^{+} \in[\delta, \lambda \delta]
$$

where $\delta=10^{-P /(2 K+3)}$, and we can express (approximately) the number of iterates $N$ in terms of the characteristic exponent $h$, the precision $P$, and the order $K$ :

$$
N \approx \frac{P}{(2 K+3) h \log _{10} \mathrm{e}}
$$

Numerical experiments show that this fit gives, for $h$ ranging in $(0,0.1]$ and $\varepsilon$ in $(-0.5,0.5)$, a maximum relative error below $4 \%$, so that it can be used to approximate the index $K$ minimizing the computer time. In order to do it, we move along the index $K$, determine the number of iterations $N$ by means of (5.10), and estimate a priori the computer time counting the total number of products and divisions performed in the algorithm. Afterwards, we choose the index $K$ associated to the smallest estimate. It should be noted that this method is very accurate: It turns out that the true optimal choice of $K$ is (at most) a ten per cent faster than our estimation.

Let us explain how, for each value of $K$, the computer time can be estimated $a$ priori. The algorithm to get the lobe area $A$ has several parts: the expansion of the local invariant curves, the Newton's method to find the pair of homoclinic orbits, the computation of the action of each homoclinic orbit, and other negligible parts. For the sake of the brevity, we shall only discuss how to estimate the time that takes the Newton's method. We can normalize the time scale in such a way that one product takes just one unit of time. Then, numerical experiments show that one division takes approximately 2.75 units of time, for large enough $P$. 

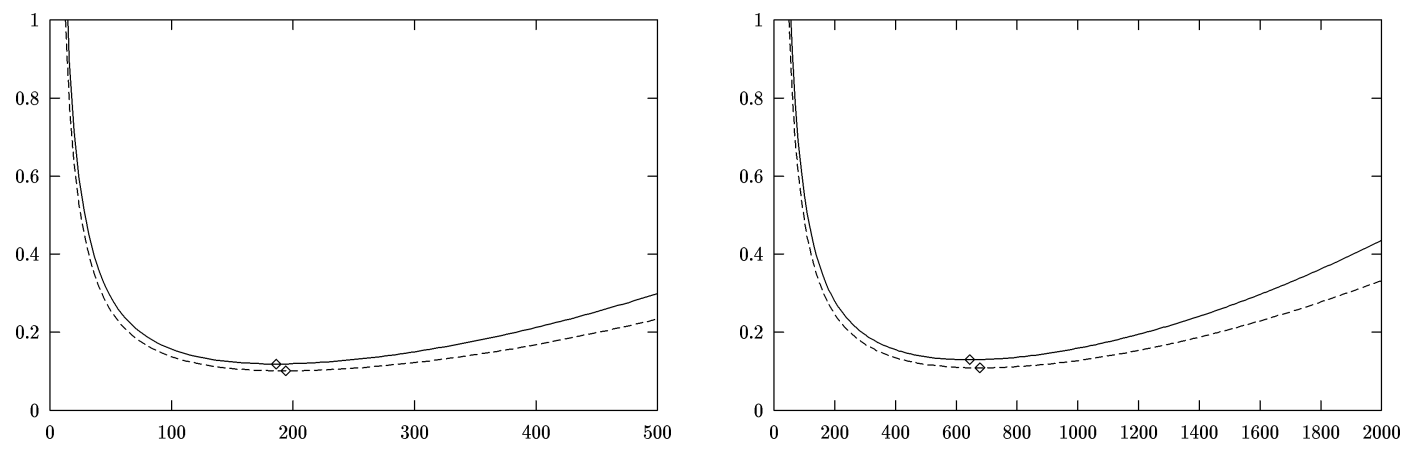

Figure 6: Estimated computer time $\mathcal{T}$ vs. the order $K$, for $\varepsilon=0.1$ and $Q=900$. The dashed lines correspond to $V^{\prime}(y)=y$ and the continuous ones to $V^{\prime}(y)=y^{3}$. The marked points correspond to the estimated optimal order. Left: $h=0.01$ and the time scale has been chosen in such a way that $10^{6}$ products with $P=1353$ decimal digits take one unit of time; the estimated optimal orders are 194 (linear case) and 186 (cubic case). Right: $h=0.001$ and the time scale has been chosen in such a way that $10^{7}$ products with $P=5211$ decimal digits take one unit of time; the estimated optimal orders are 677 (linear case) and 644 (cubic case).

Let $\#_{\times}$(respectively, $\# \div$ ) be the number of products (respectively, divisions) required to evaluate the map (2.1) together with its differential. (Of course, $\# \times \times$ and \# depend on the perturbation; for instance, in the linear case $\#_{\times}=6$ and $\# \div=1$, whereas in the cubic one $\#_{\times}=7$ and $\#_{\div}=1$.) Then, the evaluation of the function $g^{+}(r)$ given in (5.9) together with its differential takes $4 K+\left(\#_{\times}+2.75 \# \div\right) N$ units of time. The first term- $4 K$-comes from the Horner's rule to evaluate the Taylor expansions of $\sigma^{\mathrm{u}}(r)$ and $\mathrm{d} \sigma^{\mathrm{u}}(r)$. The second one- $\left(\#_{\times}+2.75 \# \div\right) N-$ is due to the computation of $F^{N}(z)$ and its differential. Therefore, the time spent during the Newton's computations is $6\left[4 K+\left(\#_{\times}+2.75 \# \div\right) N\right]$, since Newton's method takes approximately thrice what the last Newton's iteration takes, as already said, and we need compute two homoclinic orbits $(6=2 \times 3)$.

The other parts of the algorithm can be analyzed in the same way, and so one gets a closed formula $\mathcal{T}=\mathcal{T}(K)$ for the estimated computed time $\mathcal{T}$ in terms of the order $K$. Then, we take as the (estimated) optimal order the point which realizes the minimum of the function $\mathcal{T}(K)$. See figure 6 for a sample of this idea.

To end, we note that the reversibility of the map allows us to reduce the computation of homoclinic points to a one-dimensional root-finding problem, instead of a two-dimensional one. This simplifies the study, avoids stability problems and saves computer time. 


\subsection{Lobe areas}

The lobe area $A$ is the difference of actions, according to formula (5.3). Therefore, it is enough to compute the actions $W\left[\mathcal{O}^{ \pm}\right]$, but this is not so simple as applying directly formula (5.1). Let us describe briefly the problem that this simple method has. For the sake of brevity, we restrict our study to the homoclinic orbit $\mathcal{O}^{+}$.

The problem is to compute the action $W\left[\mathcal{O}^{+}\right]=\sum_{n \in \mathbb{Z}} S\left(z_{n}^{+}\right)$, where $z_{n}^{+}=F^{n}\left(z^{+}\right)$, and $z^{+}=\sigma^{\mathrm{u}}\left(r^{+}\right) \in C^{+}$is the homoclinic point previously computed. Obviously, the action must be computed to the precision $\rho=10^{-P} \leq \exp \left(-\pi^{2} / h\right)$ we are dealing with. The simplest way to get the previous infinite sum is to cut the terms with $|n|>L$, for some threshold $L$ chosen in such a way that $\left|\sum_{|n|>L} S\left(z_{n}^{+}\right)\right|<\rho$.

The generating function of the map (2.1) is

$$
S(x, y)=-x y+\mu_{0} \log \left(1+y^{2}\right)+\varepsilon V(y)
$$

From $S(z)=\mathrm{O}\left(z^{2}\right), \sigma^{\mathrm{u}, \mathrm{s}}(z)=\mathrm{O}(h z)$, and $r^{+}=\mathrm{O}(1)$, we get:

$$
S\left(z_{n}^{+}\right)= \begin{cases}S\left(\sigma^{\mathrm{u}}\left(\lambda^{-|n|} r^{+}\right)\right)=\mathrm{O}\left(h^{2} \mathrm{e}^{-2|n| h}\right) & \text { for } n \rightarrow-\infty \\ S\left(\sigma^{\mathrm{s}}\left(\lambda^{-|n|} r^{+}\right)\right)=\mathrm{O}\left(h^{2} \mathrm{e}^{-2|n| h}\right) & \text { for } n \rightarrow+\infty\end{cases}
$$

Now, we note that the lowest natural number $L$ such that $\sum_{|n|>L} h^{2} \exp (-2|n| h)<\rho \leq$ $\exp \left(-\pi^{2} / h\right)$ is at least $\mathrm{O}\left(h^{-2}\right)$. This cost of $\mathrm{O}\left(h^{-2}\right)$ evaluations of the function $S(z)$ to compute the action $W\left[\mathcal{O}^{+}\right]$becomes prohibitive for small $h$.

We proceed now to explain a better method, which requires only $\mathrm{O}\left(h^{-1}\right)$ evaluations of $S(z)$. First, the reversibility of the map allows us to reduce the computational effort to one half. Indeed, we can write the action as a difference of path integrals, see (5.2),

$$
W\left[\mathcal{O}^{+}\right]=\sum_{n \in \mathbb{Z}} S\left(z_{n}^{+}\right)=\int_{\eta^{\mathrm{u}}} y \mathrm{~d} x-\int_{\eta^{\mathrm{s}}} y \mathrm{~d} x
$$

where $\eta^{\mathrm{u}, \mathrm{s}}$ are the paths contained in the invariant curves $\mathcal{W}^{\mathrm{u}, \mathrm{s}}$ from the saddle point $z_{\infty}=(0,0)$ to the homoclinic one $z^{+}=z_{0}^{+}=\left(x^{+}, x^{+}\right) \in C^{+}$.

Since $\eta^{\mathrm{s}}=R^{+} \eta^{\mathrm{u}}$, we get

$$
W\left[\mathcal{O}^{+}\right]=\int_{\eta^{\mathrm{u}}}[y \mathrm{~d} x-x \mathrm{~d} y]=\int_{\eta^{\mathrm{u}}}[2 y \mathrm{~d} x-\mathrm{d}(x y)]=-\left(x^{+}\right)^{2}+2 \sum_{n<0} S\left(z_{n}^{+}\right),
$$

where in the last equality we have used again (5.2). To compute the last sum, we split it as follows:

$$
\sum_{n<0} S\left(z_{n}^{+}\right)=\Sigma_{1}+\Sigma_{2}, \quad \Sigma_{1}=\sum_{n<-N} S\left(z_{n}^{+}\right), \quad \Sigma_{2}=\sum_{n=-N}^{-1} S\left(z_{n}^{+}\right),
$$

where $N$ is the number of iterates that it takes to arrive at $z^{+}$from the fundamental domain in which the Taylor expansion of $\sigma^{\mathrm{u}}(r)$ holds with the required precision $\rho$. 
We write the infinite sum $\Sigma_{1}$ as a path integral along the path $\hat{\eta} \subset \mathcal{W}^{\mathrm{u}}$ from the saddle point $z_{\infty}$ to the homoclinic one $z_{-N}^{+}=\sigma^{\mathrm{u}}\left(\hat{r}^{+}\right), \hat{r}^{+} \in[\delta, \lambda \delta)$ :

$$
\Sigma_{1}=\sum_{n<-N} S\left(z_{n}^{+}\right)=\int_{\hat{\eta}} y \mathrm{~d} x=\lambda^{-1 / 2} \int_{0}^{\hat{r}^{+}} \zeta\left(\lambda^{1 / 2} r\right) \zeta^{\prime}\left(\lambda^{-1 / 2} r\right) \mathrm{d} r
$$

which can be computed with the required accuracy using the Taylor expansion of $\zeta(r)$. The second sum $\sum_{n=-N}^{-1} S\left(z_{n}^{+}\right)$is finite with only $N=\mathrm{O}\left(h^{-1}\right)$ terms, so it can be computed easily in a relatively fast way. A crucial fact to increase the efficiency of the program is the number of logarithmic evaluations required to perform this finite sum, because of the expensive multiple-precision arithmetic we are working on. Although in equation (5.11) appears a logarithm, the sum $\sum_{n=-N}^{-1} S\left(z_{n}^{+}\right)$requires just one logarithmic evaluation, since a sum of logarithms can be rewritten as the logarithm of a product.

Now we are ready to compare the two methods. The first one required at least $\mathrm{O}\left(h^{-2}\right)$ evaluations of the generating function $S(z)$, whereas the second one requires only $\mathrm{O}\left(h^{-1}\right)$ evaluations plus the computation of an integral by Taylor's method, which takes less time than the $\mathrm{O}\left(h^{-1}\right)$ evaluations of $S(z)$. Therefore, the difference is at least one order of magnitude in $h$.

To end this section, we show some values of $P, K$, and $N$ in table 2 . These results were obtained setting $\varepsilon=0.1$ and requiring $Q=900$ correct decimal digits for the lobe area. It is also displayed the true computer time $T$ in seconds (the runs have been performed on a Pentium 200 under Linux). The choice $\varepsilon=0.1$ influences very little on the quantities $P, K, N$, and $T$. In fact, $P$ does not depend on the perturbation, but only on $Q$ and $h$. As expected, the computations in the linear case- $V^{\prime}(y)=y$-are somewhat faster than in the cubic one- $V^{\prime}(y)=y^{3}-$. This is due to the following reasons: 1) The evaluation of the map $F$ with a cubic perturbation requires one more product than with the linear one, and 2) The computation of the Taylor expansion of the local invariant curve is more expensive in the cubic case, because the recurrent formulae for the Taylor coefficients require more products.

\section{$6 \quad$ Further experiments}

An interesting problem is to find an algorithm for computing the coefficients $\Theta_{n}^{\varepsilon}$ in equations (4.5), (4.6), different from the one used in this paper, which is based on the numerical continuation of lobe areas for many values of $h$, jointly with an extrapolation method. These coefficients are the unknown component in the exponentially small asymptotic formula for the splitting size. For some celebrated standard-like maps, similar quantities (like the Lazutkin's constant $\omega_{0}=1118.827706 \ldots$ for the standard map) have been defined by means of nonlinear parameterless problems which only can be solved numerically [GLT91, HM93, GLS94, Sur94, Che95, Nik95, Tre96]. It would be useful to find such a problem for $\Theta_{n}^{\varepsilon}$, since the absence of parameters makes easier its resolution. 
Table 2: Values of $P$ (decimals digits in the multiple-precision arithmetic), $K$ (local order), $N$ (iterations), and $T$ (computing time in seconds), for $Q=900$ (decimal digits required for the lobe area) and $\varepsilon=0.1$.

\begin{tabular}{c|c|c|c|c|c|c|c}
\multicolumn{2}{c|}{} & \multicolumn{3}{c|}{$V^{\prime}(y)=y$} & \multicolumn{3}{c}{$V^{\prime}(y)=y^{3}$} \\
\hline$h$ & $P$ & $K$ & $N$ & $T$ & $K$ & $N$ & $T$ \\
\hline 0.010 & 1353 & 194 & 800 & 1437 & 186 & 834 & 1371 \\
0.009 & 1401 & 204 & 876 & 1652 & 195 & 916 & 1879 \\
0.008 & 1459 & 215 & 974 & 1935 & 206 & 1016 & 2205 \\
0.007 & 1536 & 230 & 1096 & 2369 & 220 & 1145 & 2736 \\
0.006 & 1637 & 248 & 1264 & 3038 & 237 & 1322 & 3379 \\
0.005 & 1782 & 271 & 1511 & 4134 & 259 & 1581 & 4643 \\
0.004 & 1994 & 305 & 1879 & 6195 & 291 & 1969 & 7091 \\
0.003 & 2350 & 356 & 2532 & 11154 & 339 & 2658 & 12613 \\
0.002 & 3068 & 447 & 3951 & 31614 & 426 & 4145 & 36389 \\
0.001 & 5211 & 677 & 8869 & 207007 & 644 & 9321 & 240530
\end{tabular}

To perform a similar study for (large and/or small) perturbations of other integrable maps is the most natural continuation of this work.

As a first example, we mention the integrable standard-like maps given by Suris in [Sur89]. (The McMillan map is a particular case of the Suris maps.) For instance, [LM96] contains a exponentially small Melnikov prediction in the characteristic exponent for the lobe area in a perturbed trigonometric Suris map, together with a numerical study in double-precision of its validity. It would be interesting to work out these computations in multiple-precision.

As a second example, we mention the twist maps associated to the perturbations of elliptic billiards. The papers [LT93, Tab94, DR96, Lom96, Lev97] contain exponentially small predictions for the splitting size when the eccentricity is small, that is, when the unperturbed ellipse is near to a circle. The numerical experiments can be specially helpful, since there is still a lack of analytical results. However, it is worth noting that the numerical study of billiards is somewhat harder than the one performed here. This has to do with the fact that the twist maps associated to billiards have not explicit expressions, since they are defined implicitly by means of their generating functions. Therefore, the evaluation of the map is more expensive: one needs to solve implicit equations with trigonometric terms.

Volume-preserving maps form the third example where a detailed numerical analysis would be interesting. In $[\mathrm{AC} * 92, \mathrm{RK} * 93]$, one can find several families of volumepreserving maps, depending on a small parameter $h$, such that the splitting distance between certain invariant curves behaves with respect to $h$ as in (1.2). The arguments in these papers are semi-analytical. It would be interesting to study numerically the asymptotic behavior of these distances. Maybe, a behavior like (1.3) or even (1.4) may 
be established, if a multiple-precision arithmetic is used.

As a last application, we consider the symplectic high-dimensional case. In [DR97b] we obtained exponentially small asymptotic predictions via Melnikov methods for some perturbations of the McLachlan map (a high-dimensional generalization of the McMillan map here studied). The computations in the high-dimensional case must be performed very carefully. The main difficulties associated to the increase of dimension are the computation of the invariant manifolds, which takes much more time than in the planar case, and the sensitive dependence of Newton's method on the initial approximation. Following [Tab95], we suggest a way to overcome these problems. The first difficulty can be soften using the Lagrangian property of the invariant manifolds of symplectic maps, which can be written as graphs of gradients of a scalar function called generating function of the manifold. The idea is to compute the Taylor expansion of such generating functions instead of dealing with the invariant manifolds. To overcome the sensitive dependence of Newton's method on the initial approximation, one can use first the method developed in [Tab95] to find homoclinic points, based on the computation of critical points of a scalar function, usually a more robust problem. Then, one can refine the homoclinic point using the Newton's method (or a quasi-Newton method), which converges faster.

Finally, we want to mention an outstanding conjecture, due to C. Simó, on the asymptotic behavior of the splitting size for some area-preserving maps like the standard map, the Hénon map, the twist map, and the perturbed McMillan map studied here. Roughly speaking, this conjecture claims that

$$
\text { splitting size }=\sum_{m \geq 1} h^{\gamma_{m}} \mathrm{e}^{-m \beta / h} \Theta_{m}(h), \quad \Theta_{m}(h) \text { Gevrey-1 and } \Theta_{m}(0) \neq 0,
$$

that is, smaller exponentials must be added to (1.4) in order to get a more exact formula. These exponentials do not play any rôle for "small" values of $h$, but they become significant for "larger" ones. There are strong reasons for believing that (6.1) holds, but nowadays there is a lack of analysis and computer power to tackle this conjecture. We hope that this will be a stimulating challenge for some of the readers.

\section{Acknowledgments}

This work has been partially supported by the EC grant ERBCHRXCT-940460. Research by A. Delshams is also supported by the Spanish grant DGICYT PB94-0215 and the Catalan grant CIRIT 1996SGR-00105. Research by R. Ramírez-Ros is also supported by the U.P.C. grant PR9409. Both authors wish to express their appreciation to R. de la Llave and C. Simó for several private communications, and to À. Jorba for his help in the design of the multiple-precision routines. It is also a pleasure to thank A. Benseny and E. Fontich for very stimulating discussions and fruitful remarks. This work was finished while one of the authors (A.D.), was a visitor at the Institute for Mathematics and Its Applications in Minneapolis, for whose hospitality he is very grateful. 


\section{References}

[AC*92] C. Amick, S.C.E. Ching, L.P. Kadanoff, and V. Rom-Kedar. Beyond all orders: Singular perturbations in a mapping. J. Nonlinear Sci., 2:9-67, 1992.

[BK97] W.-J. Beyn and J.-M. Kleinkauf. The numerical computation of homoclinic orbits for maps. SIAM J. Numer. Anal., 34(3):1207-1236, 1997.

[BO93] A. Benseny and C. Olivé. High precision angles between invariant manifolds for rapidly forced Hamiltonian systems. In C. Perelló, C. Simó, and J. Solà-Morales, editors, International Conference on Differential Equations (Equa $\frac{\partial i}{\partial t} f f$ 91), pages 315319. Held in Barcelona, 26-31 August, 1991. World Scientific, Singapore, 1993.

[Che95] V.L. Chernov. Separatrix splitting for the Hénon map: a resurgence approach. Math. Preprints Series 188, Univ. Barcelona, November 1995.

[DR96] A. Delshams and R. Ramírez-Ros. Poincaré-Melnikov-Arnold method for analytic planar maps. Nonlinearity, 9(1):1-26, 1996.

[DR97a] A. Delshams and R. Ramírez-Ros. Exponentially small splitting of separatrices for perturbed integrable standard-like maps. mp_arc@math.utexas.edu, preprint 97160, March 1997. To appear in J. Nonlinear Sci.

[DR97b] A. Delshams and R. Ramírez-Ros. Melnikov potential for exact symplectic maps. Preprint 97-150, mp_arc@math .utexas . edu, March 1997. To appear in Comm. Math. Phys.

[DRS97] A. Delshams, R. Ramírez-Ros, and T.M. Seara. Splitting of separatrices in Hamiltonian systems and symplectic maps. In C. Simó, editor, Hamiltonian Systems with Three or More Degrees of Freedom, NATO Adv. Sci. Inst. Ser. C Math. Phys. Sci. Held in S'Agaró, Spain, 19-30 June 1995. Kluwer Acad. Publ., Dordrecht, Holland, to appear in 1997.

[DS97] A. Delshams and T.M. Seara. Splitting of separatrices in Hamiltonian systems with one and a half degrees of freedom. Math. Phys. Electron. J., 3(4):1-40, 1997.

[Eas91] R.W. Easton. Transport through chaos. Nonlinearity, 4:583-590, 1991.

[Fon89] E. Fontich. On the existence of homoclinic points for conservative diffeomorphisms. In C. Alsina, J. Llibre, Ch. Mira, C. Simó, G. Targonski, and R. Thibault, editors, European Conference on Iteration Theory (ECIT 87), pages 189-197. Held in Caldes de Malavella, Spain, 20-26 September, 1987. World Scientific, Singapore, 1989.

[Fon95] E. Fontich. Rapidly forced planar vector fields and splitting of separatrices. $J$. Differential Equations, 119(2):310-335, 1995.

[FS90] E. Fontich and C. Simó. The splitting of separatrices for analytic diffeomorphisms. Ergodic Theory Dynam. Systems, 10:295-318, 1990.

[FS96] B. Fiedler and J. Scheurle. Discretization of homoclinic orbits, rapid forcing and "invisible" chaos. Mem. Amer. Math. Soc., 119(570), 1996. viii + 79 pp. 
[Gel96] V.G. Gelfreich. Conjugation to a shift and the splitting of invariant manifolds. Appl. Math. (Warsaw), 24(2):127-140, 1996.

[GLS94] V.G. Gelfreich, V.F. Lazutkin, and N.V. Svanidze. A refined formula for the separatrix splitting for the standard map. Phys. D, 71:82-101, 1994.

[GLT91] V.G. Gelfreich, V.F. Lazutkin, and M.B. Tabanov. Exponentially small splitting in Hamiltonian systems. Chaos, 1(2):137-142, 1991.

[HM93] V. Hakim and K. Mallick. Exponentially small splitting of separatrices, matching in the complex plane and Borel summation. Nonlinearity, 6:57-70, 1993.

[Knu69] D.E. Knuth. The art of computer programming. Vol. 2: Seminumerical algorithms. Addison-Wesley Publishing Co., Reading, Mass.-London-Don Mills, Ont, 1969.

[Laz84] V.F. Lazutkin. Splitting of separatrices for the Chirikov's standard map. Preprint VINITI No. 6372-84 (in Russian), 1984.

[Lev97] P. Levallois. Calcul d'une fonction de Melnikov et de ses zeros pour une perturbation algébrique du billard elliptique. Ergodic Theory Dynam. Systems, 17:435-444, 1997.

[LM96] H.E. Lomelí and J.D. Meiss. Heteroclinic orbits, and transport in a perturbed integrable standard map. Preprint 96-77, mp_arc@math .utexas .edu, February 1996.

[Lom96] H.E. Lomelí. Perturbations of elliptic billiards. Phys. D, 99:59-80, 1996.

[LST89] V.F. Lazutkin, I.G. Schachmannski, and M.B. Tabanov. Splitting of separatrices for standard and semistandard mappings. Phys. D, 40:235-248, 1989.

[LT93] P. Levallois and M.B. Tabanov. Séparation des séparatrices du billard elliptique pour une perturbation algébrique et symétrique de l'ellipse. C. R. Acad. Sci. Paris Sér. I Math., 316:589-592, 1993.

[McM71] E.M. McMillan. A problem in the stability of periodic systems. In E. Brittin and H. Odabasi, editors, Topics in modern physics, a tribute to E.V. Condon, pages 219-244. Colorado Assoc. Univ. Press, Boulder, CO, 1971.

[Mei92] J.D. Meiss. Symplectic maps, variational principles, and transport. Rev. Mod. Phys., 64(3):795-848, 1992.

[MMP84] R.S. MacKay, J.D. Meiss, and I.C. Percival. Transport in Hamiltonian systems. Phys. D, 13:55-81, 1984.

[MMP87] R.S. MacKay, J.D. Meiss, and I.C. Percival. Resonances in area preserving maps. Phys. D, 27:1-20, 1987.

[Nei84] A.I. Neishtadt. The separation of motions in systems with rapidly rotating phase. J. Appl. Math. Mech., 48(2):133-139, 1984.

[Nik95] A. Nikitin. Separatrix splitting via résurgence for the 'twist map'. A method for the calculation of the coefficients of the splitting formula. Math. Preprints Series 184, Univ. Barcelona, November 1995. 
[RK*93] V. Rom-Kedar, L.P. Kadanoff, S.C.E. Ching, and C. Amick. The break-up of a heteroclinic connection in a volume preserving mapping. Phys. D, 62:51-65, 1993.

[RS96] J.-P. Ramis and R. Schäfke. Gevrey separation of fast and slow variables. Nonlinearity, 9:353-384, 1996.

[Sau95] D. Sauzin. Résurgence paramétrique et exponentielle petitese de l'ecart des séparatrices du pendule rapidament forcé. Ann. Inst. Fourier (Grenoble), 45(2):453$511,1995$.

[Sim90] C. Simó. On the analytical and numerical approximation of invariant manifolds. In D. Benest and C. Froeschlé, editors, Les méthodes modernes de la mécanique céleste, pages 285-329. Held in Goutelas, 1989. Editions Frontières, Gif-sur-Yvette, 1990.

[SMH91] J. Scheurle, J. Marsden, and P.J. Holmes. Exponentially small estimate for separatrix splittings. In H. Segur, S. Tanveer, and H. Levine, editors, Asymptotics beyond All Orders, pages 187-195. NATO Adv. Sci. Inst. Ser. B Phys., vol. 284, Plenum, New York, 1991.

[Sur89] Y.B. Suris. Integrable mappings of the standard type. Functional Anal. Appl., 23:74-76, 1989.

[Sur94] Y.B. Suris. On the complex separatrices of some standard-like maps. Nonlinearity, 7:1225-1236, 1994.

[Tab94] M.B. Tabanov. Separatrices splitting for Birkhoff's billiard in a symmetric convex domain, close to an ellipse. Chaos, 4(4):595-606, 1994.

[Tab95] E. Tabacman. Variational computation of homoclinic orbits for twist maps. Phys. $D, 85: 548-562,1995$.

[Tre96] D.V. Treschev. An averaging method for Hamiltonian systems, exponentially close to integrable ones. Chaos, 6(1):6-14, 1996.

[Wei73] A. Weinstein. Lagrangian submanifolds and Hamiltonian systems. Ann. of Math. (2), 98:377-410, 1973.

\section{Internet access:}

All the authors' quoted preprints, as well as the code for the programs used in this paper, are available at http://www-ma1.upc.es in the Preprints pages, or at ftp://ftp-ma1.upc.es, in the pub/preprints directory.

E-mail addresses of the authors: amadeu@ma1.upc.es, rafael@tere.upc.es. 